University of Louisville

ThinkIR: The University of Louisville's Institutional Repository

Electronic Theses and Dissertations

$5-2020$

\title{
Mathematical characterization of a microfluidic ultrasound driven transfection device.
}

Chris J. Holton

University of Louisville

Follow this and additional works at: https://ir.library.louisville.edu/etd

Part of the Biomedical Engineering and Bioengineering Commons

\section{Recommended Citation}

Holton, Chris J., "Mathematical characterization of a microfluidic ultrasound driven transfection device." (2020). Electronic Theses and Dissertations. Paper 3348.

https://doi.org/10.18297/etd/3348

This Master's Thesis is brought to you for free and open access by ThinkIR: The University of Louisville's Institutional Repository. It has been accepted for inclusion in Electronic Theses and Dissertations by an authorized administrator of ThinkIR: The University of Louisville's Institutional Repository. This title appears here courtesy of the author, who has retained all other copyrights. For more information, please contact thinkir@louisville.edu. 
MATHEMATICAL CHARACTERIZATION OF A MICROFLUIDIC ULTRASOUND DRIVEN TRANSFECTION DEVICE

\title{
By
}

Christopher James Holton

B.S. Bioengineering, University of Louisville, May 2019

\author{
A Thesis \\ Submitted to the Faculty of the \\ University of Louisville \\ J.B. Speed School of Engineering \\ as Partial Fulfillment of the Requirements \\ for the Professional Degree
}

MASTER OF ENGINEERING

Department of Bioengineering

May 2020 


\section{ACKNOWLEDGMENTS}

First, I would like to thank Dr. Kopechek for agreeing to advise me on this thesis and allowing me to be a part of his research. You have been extremely helpful and instrumental in developing the following work. I would like to thank you for your patience and guidance not only throughout this thesis, but during the classes of yours I took and during my senior project as well. Thank you for funding me as a research assistant and allowing me to be a part of the entrepreneurial development of the device during the ICorps program. You are one of the most kind, smart, and hardest working people I know and I am so grateful to have had you as a mentor. I am so optimistic about the future of this research and the thousands of lives it will impact in a positive way. I wish you and your family the best of luck in the future.

Next, I would like to thank my committee members, Dr. Tommy Roussel and Dr. Kavitha Yaddanapudi, for agreeing to be a part of my defense committee and for their time spent in reviewing this thesis. Thank you for being available for discussion and taking time to coordinate with me.

I would also like to thank the researchers in Dr. Kopechek's lab who came before me or are still there. Specifically, Connor Centner, Emily Murphy, Mariah Priddy, and Erik Black, thank you for any assistance you knowingly or unknowingly provided me during the development of this thesis. 
Lastly, I would like to thank my parents and brothers. Your all's support has been unwavering and I wouldn't be anywhere close to where I am today without everything you all have done for me. I can't thank you enough and only hope I can repay you with the same support and love in the future. 


\begin{abstract}
The objective of this thesis is to develop a mathematical model characterizing the behavior of a microfluidic sonoporation device in order to understand how standing wave conditions influence molecular delivery to cells and determine whether the model predicts device performance. A prior model based on an ultrasonic separation cell that uses standing waves to separate particles is adapted for translation to the microfluidic device. This study generates data on acoustic pressure profiles across the cell as well as identifying optimal driving frequencies. This model is validated and the equations and methods for developing this model are translated to the microfluidic device. An investigation into the variation of cell layer parameters and driving frequencies is conducted to understand their influence on acoustic pressure profiles and resonant frequencies across the cell. These data are compared to experimental trials which measure cellular uptake of fluorescence when driven through the microfluidic device exposed to different ultrasound frequencies. Results suggest that the $6 \mathrm{MHz}$ driving frequency generates the largest pressure profile across the cell but does not correlate with high molecular delivery efficiency during experimental trials. Additional conclusions regarding the acoustic pressure profile dependency on density, thickness, and speed of sound within the layers show a significant effect for specific frequencies. The large variation in results for differing material and geometric parameters shows the need for
\end{abstract}


further refinement of these parameters for the laboratory device. Once additional experimental trials are conducted, more iterations of the model are tested, and cell parameters are more precisely determined, the translated model can be used for extensive characterization of acoustic pressure profiles across the cell for future design iterations of the device. 


\section{TABLE OF CONTENTS}

PAGE

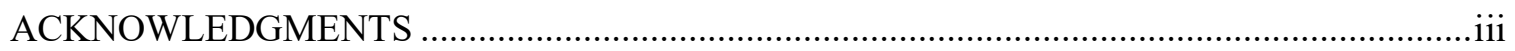

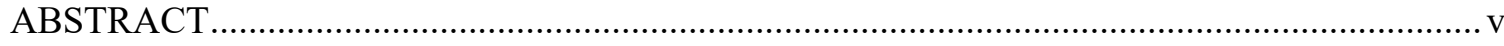

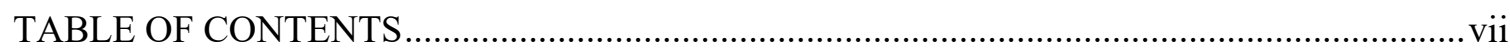

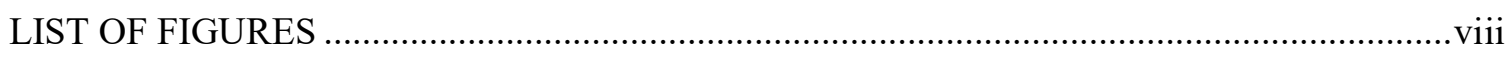

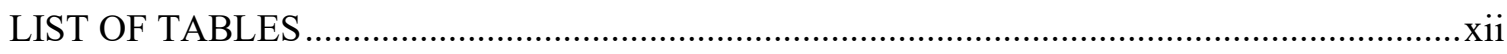

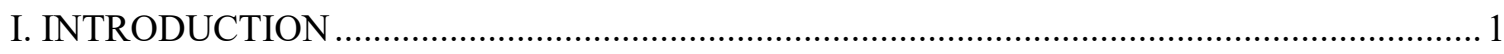

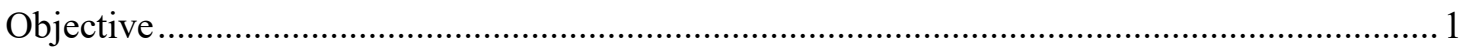

Overview of Transfection and Molecular Delivery ............................................................. 1

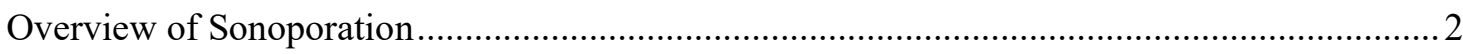

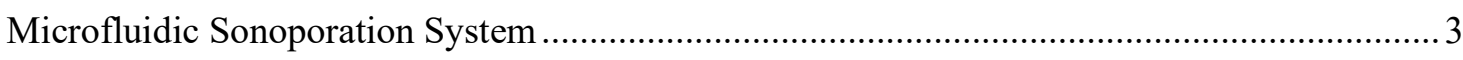

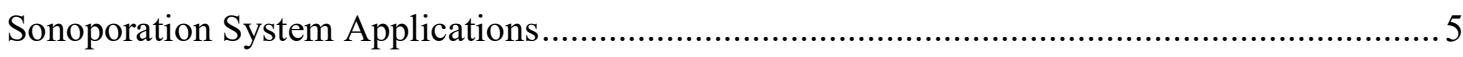

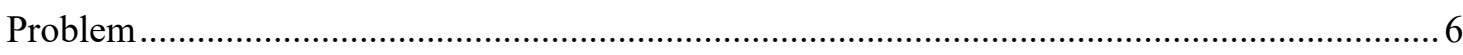

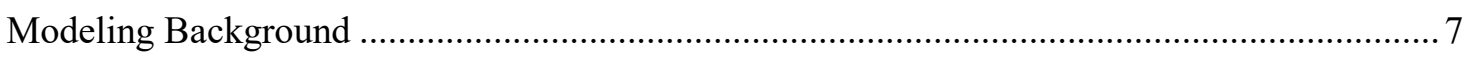

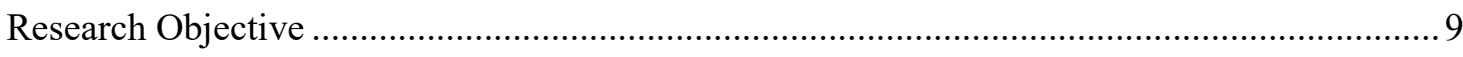

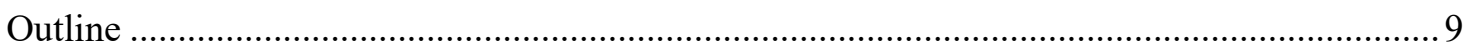

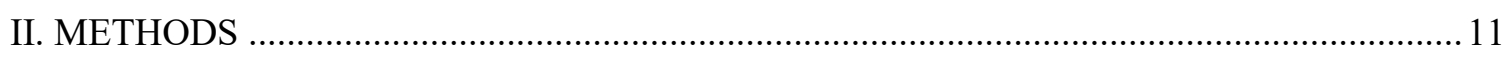

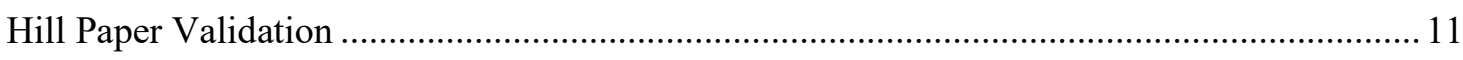

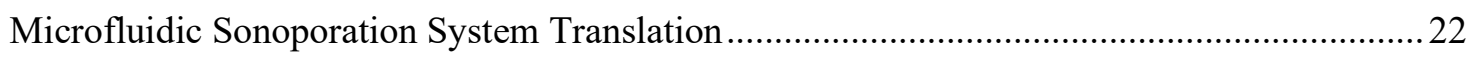

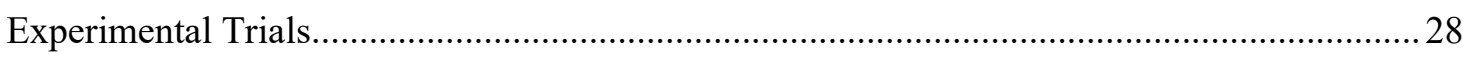

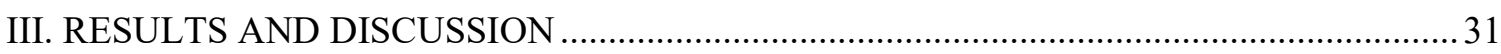

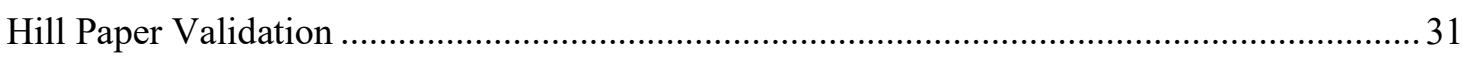

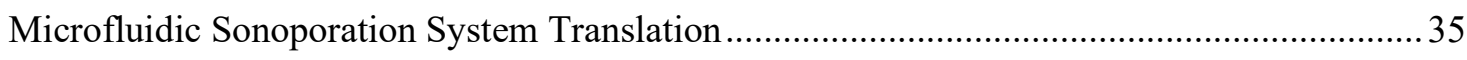

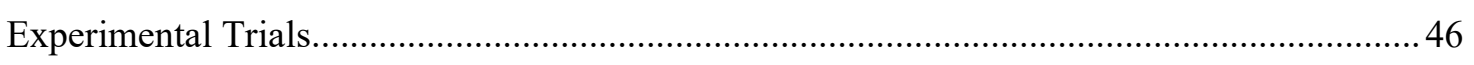

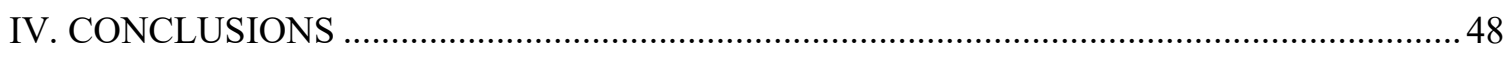

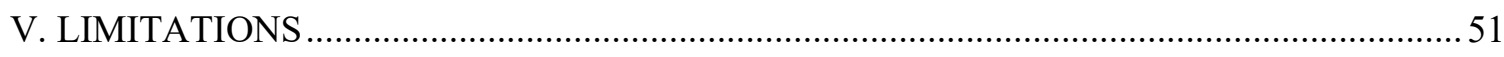

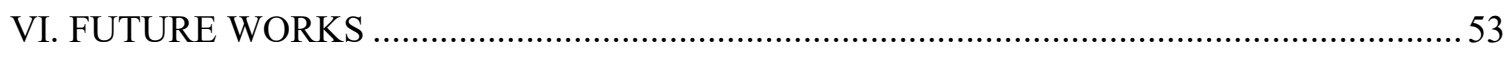

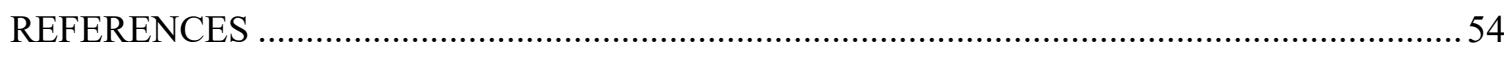

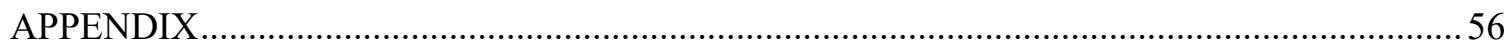




\section{LIST OF FIGURES}

PAGE

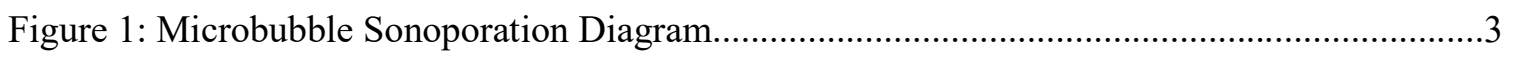

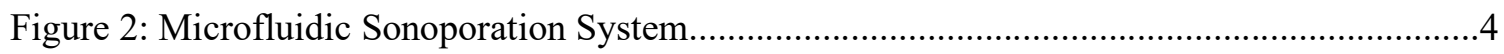

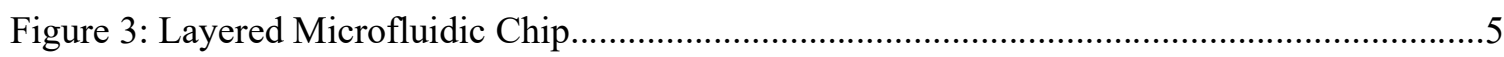

Figure 4: Transducer Equivalent Resonance Circuit..............................................................

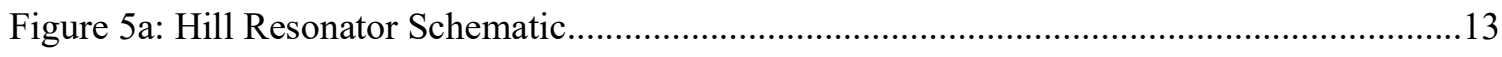

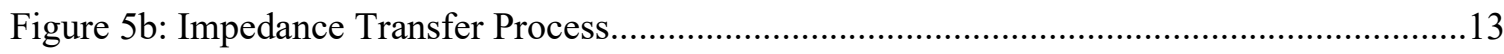

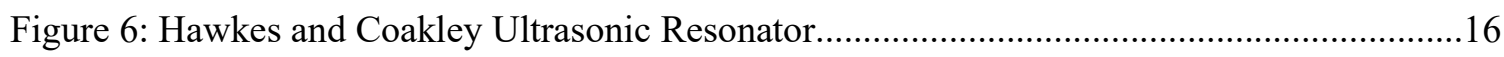

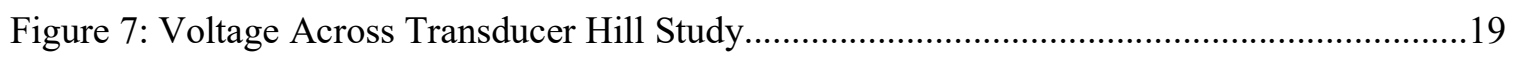

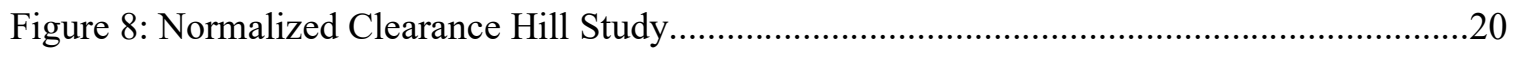

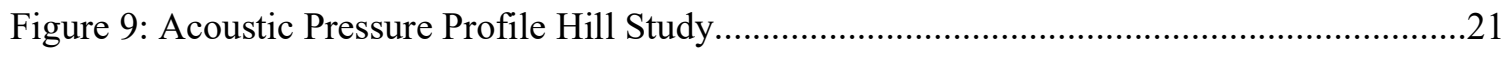

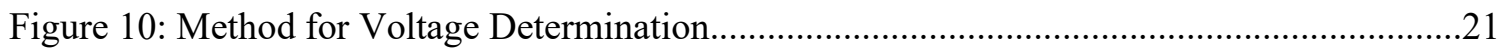

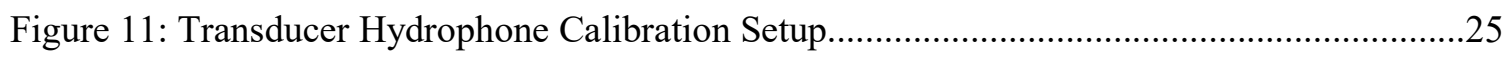

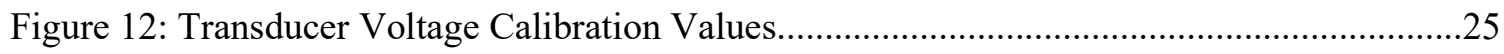

Figure 13a: Replicated Pressure Profile Hill Transformation Ratio of 1.....................................31

Figure 13b: Replicated Pressure Profile Hill Left Boundary Values.............................................31

Figure 14: Replicated Pressure Profile with Specific Transformation Ratios................................32

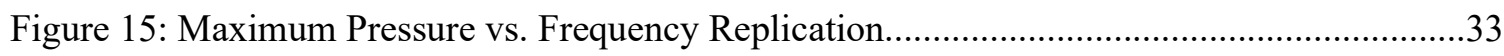

Figure 16: Normalized Maximum Pressure vs. Frequency Replication........................................34

Figure 17: Pressure vs. Position Minimum Silicone Density Water Layer...................................35

Figure 18: Pressure vs. Position Minimum Silicone Density Blood Layer....................................35

Figure 19: Pressure vs. Position Average Silicone Density Water Layer.......................................35 
Figure 20: Pressure vs. Position Average Silicone Density Blood Layer.......................................35

Figure 21: Pressure vs. Position Maximum Silicone Density Water Layer...................................36

Figure 22: Pressure vs. Position Maximum Silicone Density Blood Layer....................................36

Figure 23: Pressure vs. Position Minimum Silicone Speed of Sound Water Layer........................37

Figure 24: Pressure vs. Position Minimum Silicone Speed of Sound Blood Layer........................37

Figure 25: Pressure vs. Position Average Silicone Speed of Sound Water Layer...........................37

Figure 26: Pressure vs. Position Average Silicone Speed of Sound Blood Layer...........................37

Figure 27: Pressure vs. Position Maximum Silicone Speed of Sound Water Layer........................38

Figure 28: Pressure vs. Position Maximum Silicone Speed of Sound Blood Layer........................38

Figure 29: Pressure vs. Position Minimum PDMS Speed of Sound Water Layer.........................39

Figure 30: Pressure vs. Position Minimum PDMS Speed of Sound Blood Layer.........................39

Figure 31: Pressure vs. Position Average PDMS Speed of Sound Water Layer...........................39

Figure 32: Pressure vs. Position Average PDMS Speed of Sound Blood Layer...........................39

Figure 33: Pressure vs. Position Maximum PDMS Speed of Sound Water Layer.........................40

Figure 34: Pressure vs. Position Maximum PDMS Speed of Sound Blood Layer..........................40

Figure 35: Pressure vs. Position Half Silicone Thickness Water Layer........................................41

Figure 36: Pressure vs. Position Double Silicone Thickness Water Layer....................................41

Figure 37: Pressure vs. Position Normal Silicone Thickness Water Layer...................................42

Figure 38: Pressure vs. Position Half Glass Thickness Water Layer............................................42

Figure 39: Pressure vs. Position Double Glass Thickness Water Layer.......................................42

Figure 40: Pressure vs. Position Normal Glass Thickness Water Layer........................................43

Figure 41: Pressure vs. Position Half PDMS Thickness Water Layer........................................43

Figure 42: Pressure vs. Position Double PDMS Thickness Water Layer......................................43

Figure 43: Pressure vs. Position Normal Glass Thickness Water Layer......................................44

Figure 44: Maximum Pressure vs. Frequency Water Layer.......................................................45

Figure 45: Maximum Pressure vs. Frequency Blood Layer...........................................................45 
Figure 46: Normalized Maximum Pressure vs. Frequency Water Layer.....................................46

Figure 47: Normalized Maximum Pressure vs. Frequency Blood Layer.......................................46

Figure 48: Experimental Trial Relative Fluorescence Frequency Dependency............................47 


\section{LIST OF TABLES}

PAGE

Table 1: Material and geometric parameters for Hill layered cell ............................................. 17

Table 2:Additional values for developing resonator model ...................................................... 18

Table 3: Material and geometric parameters for the microfluidic device. Fluid channel properties

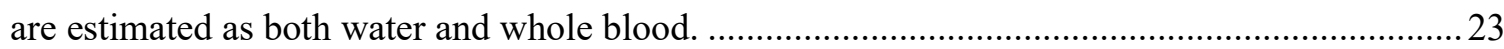

Table 4: Electrical values for the PZT transducer and the driving frequencies as well as fluid

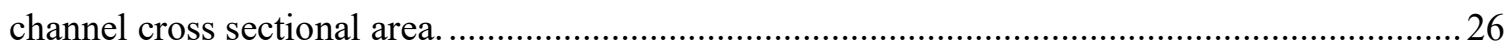

Table 5: Specific transformation ratios for each driving frequency from Figure 13. Values to

calculate transformation ratio taken from Figure $13 \mathrm{~b}$ and left boundary values of Figure $9 \ldots \ldots . . .32$ 


\section{INTRODUCTION}

\section{Objective}

The objective of this thesis is to develop a mathematical model to characterize the behavior of a microfluidic sonoporation device in an effort to understand how standing wave conditions influence molecular delivery to cells and determine whether the model predicts device performance.

\section{Overview of Transfection and Molecular Delivery}

Cell transfection is a technique in which genetic material is delivered into cells to express specific proteins. These proteins are used to treat diseases by targeting specific, disease-causing cells. Molecular delivery is similar to transfection, but small molecules are delivered to the cell as opposed to genetic material. Originally, the most prevalent method of transfection was viral transfection, known as transduction [1]. This method uses the inherent ability of viruses to deliver genetic material to cells and has been shown to be highly efficient in this task [1]. However, this method also has issues with specificity, safety, and manufacturing among others $[1,2]$. In light of these limitations, non-viral methods were developed which employ different techniques to deliver genetic material inside the cell. Reagent based transfection techniques package genes in 
liposomes, proteins, or polymers which penetrate the cell membrane [2]. This method has been shown to have low toxicity and induces fewer immune responses, but it also has issues with efficiency and optimization for different cell types [1,2]. Electroporation is another non-viral transfection method which uses electrical pulses to open temporary pores in cell membranes for gene delivery [2]. This method has been shown to work for both transient and stable transfection objectives for different cell types. However, it also suffers from issues with efficiency when scaled up and issues with throughput when it is scaled down [1,2]. Additionally, electroporation requires specific buffers that conduct current efficiently and some cells do not survive well in those conditions [2]. Another disadvantage of electroporation is that it relies on passive diffusion of molecules into the cells. Electroporation opens pores in cells but it does not have an active mechanism for transporting nearby molecules into the cell [2]. The main focus of the experimental portion of this study is on the molecular delivery of small fluorescent molecules to red blood cells, but it should be stated that transfection applications are also important for future work.

An emerging method of non-viral transfection which aims to combat all these issues is sonoporation. Sonoporation does not require buffers or viruses or large amounts of reagent and is capable of developing transient pores in cells while inducing active diffusion through a phenomenon known as microstreaming.

\section{Overview of Sonoporation}

Sonoporation is a method which uses acoustic waves to increase the permeability of the cell membrane to genetic material or other biomolecules [3]. This technique (Figure 
1) employs the use of microbubbles to improve efficiency, which are lipid-shelled, gasfilled spheres that often contain the molecular or genetic material for insertion $[2,3]$. When exposed to a rapid change in pressure from ultrasonic pulses these microbubbles oscillate, rapidly expand and compress, and eventually rupture resulting in the generation of a shock wave [3]. This phenomenon, known as inertial cavitation, creates transient pores in nearby cell membranes and induces a microjet (microstreaming) which can drive the genetic material inside the cell [3]. These pores reseal quickly which makes this a promising technique for a wide range of in-vivo targeted transfection treatments [3].

Similar to reagent-based transfection and electroporation, this method can suffer from issues with efficiency and optimization of parameters for different cell types and throughput objectives. Development of ultrasound-integrated microfluidic devices could address those limitations.

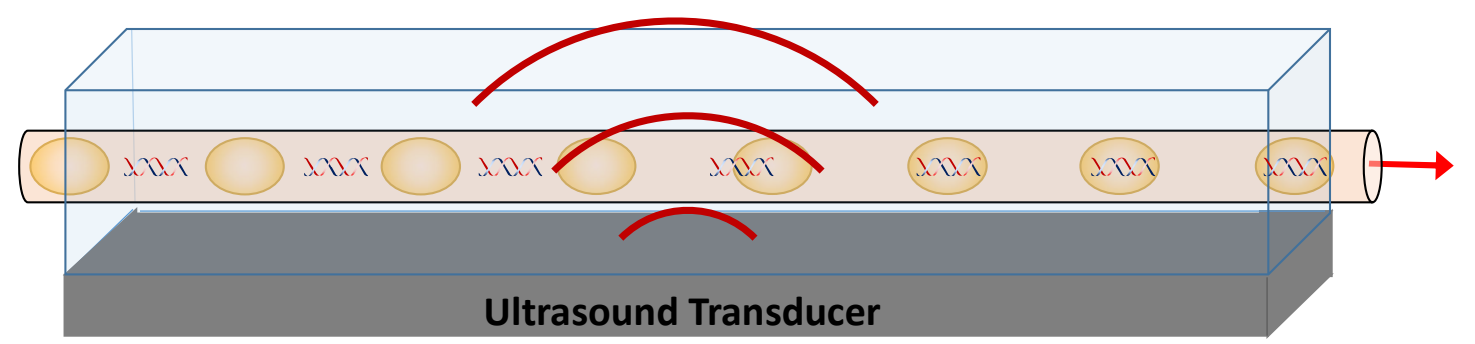

Figure 1: An ultrasonic transducer sends pulses through a solution of microbubbles, cells, and genetic material. The pulses cause the bubbles to oscillate and collapse resulting in a shock wave that drives genetic material into the cells.

\section{Microfluidic Sonoporation System}

One system that utilizes the microbubble enhanced sonoporation technique is the system currently in development in Dr. Jonathan Kopechek's laboratory at the University of Louisville. The first iteration of this device used a clinical ultrasound probe directed at a conical tube in a water tank to treat cells with ultrasonic waves. This setup proved to be 
inefficient as many of the cells in the bulk setup were shielded by other cells and the ultrasonic pulses had attenuated too much before reaching them to provide effective treatment [2]. Due to this, a more targeted, smaller scale version of the device was created which uses a microfluidic setup. The current iteration of the system (Figure 2) uses a PDMS (polydimethylsiloxane) or 3D printed chip etched with a microfluidic channel to focus the area of inertial cavitation and transfection. Connected to this chip is an ultrasonic transducer which is driven by a microcontroller to deliver ultrasonic pulses at specified frequencies and amplitudes. The fluid system is driven by syringe pumps running at a specified flow rate, which force the microbubble-cell solution through tubing that connects to the microfluidic channel. As the cell solution passes through the microchannel etching, ultrasonic pulses from the lead zirconate titanate (PZT) transducer increase cell permeability, cause the microbubbles to burst, and the genetic material to

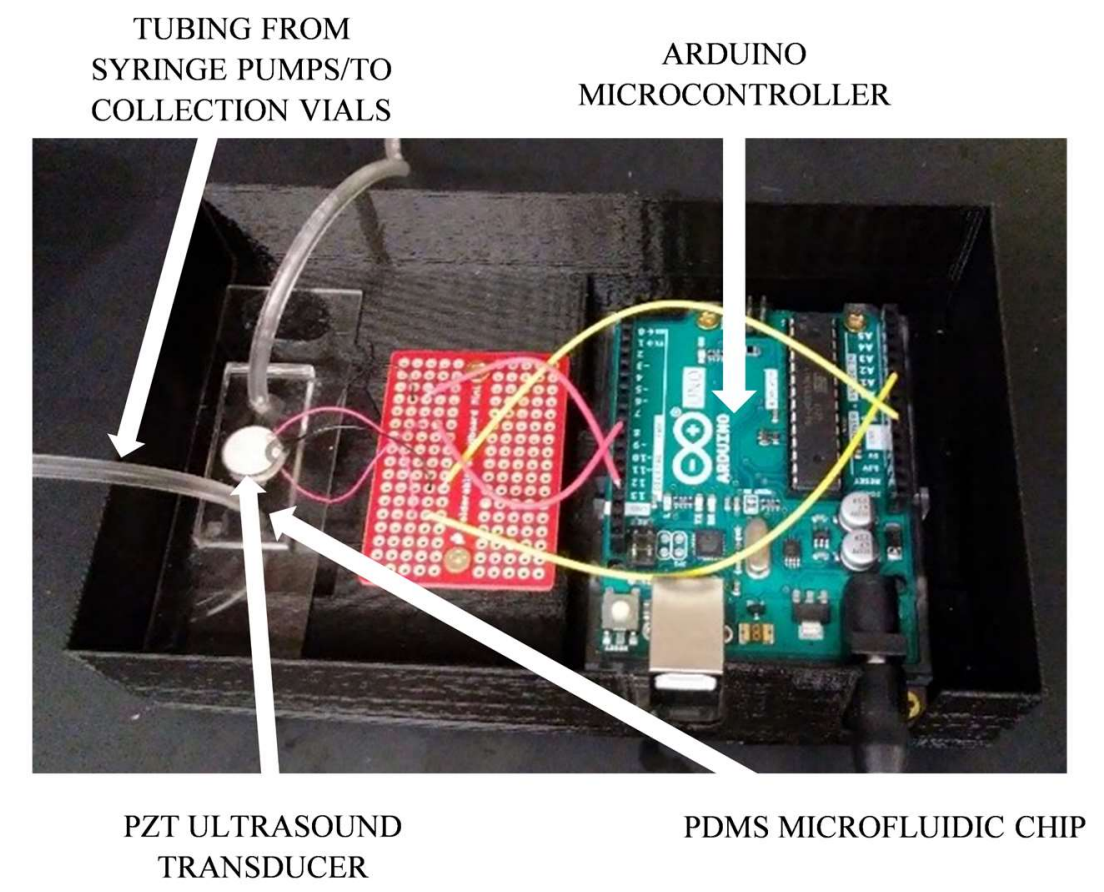

Figure 2: Microfluidic sonoporation system in Dr. Kopechek's lab at the University of Louisville. Elements are encased in a 3D printed box. 
enter the cells. This treated solution of cells is collected in vials for post-processing and viability assessment.

The PDMS-based version of the microfluidic chip (Figure 3) contains multiple layers. The PZT transducer oscillates against a layer of silicone. This layer of silicone is connected to a glass slide which is laid over the top of the microfluidic channel. The microfluidic channel is etched into a layer of PDMS. This image is not to scale, but the size of the transducer should be larger than the width of the microchannel to ensure longitudinal waves are the dominant acoustical characteristics within the channel.

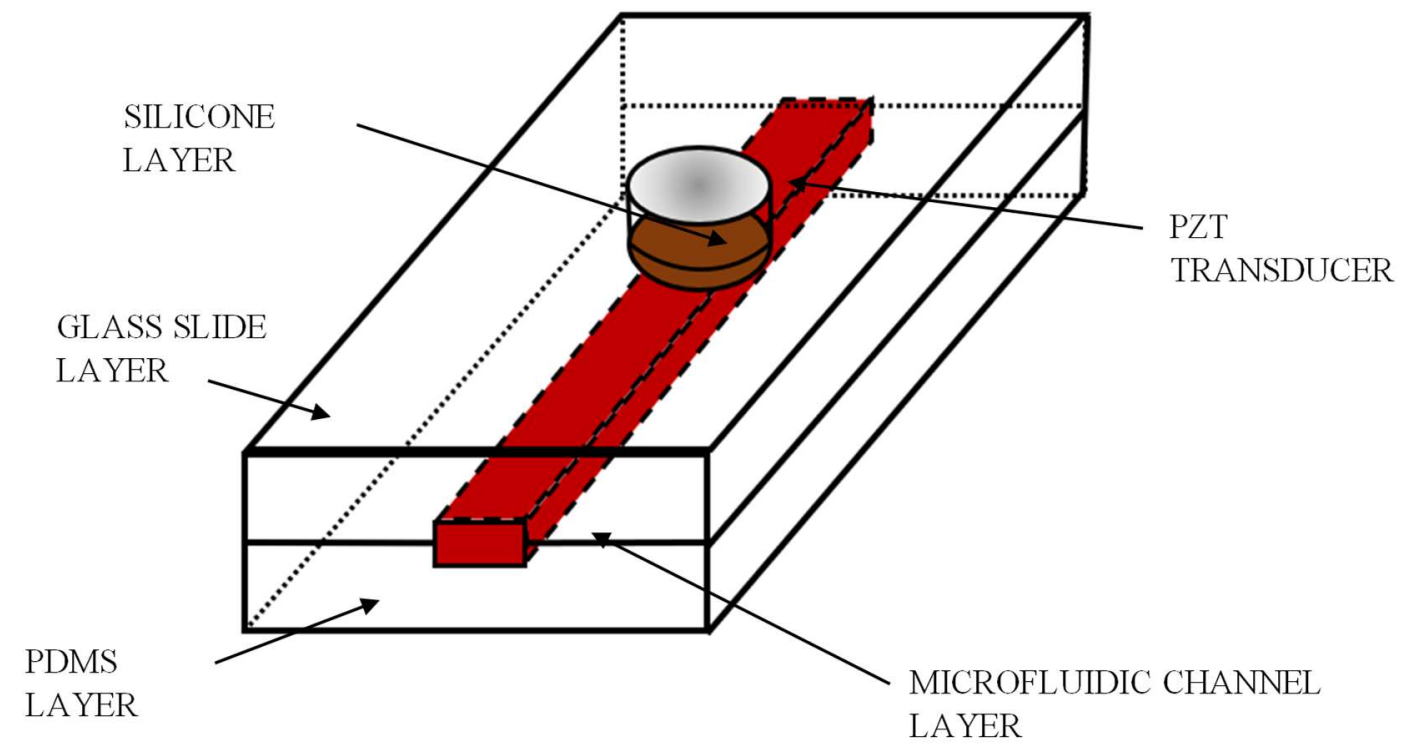

Figure 3: Layered microfluidic chip. PDMS based version

\section{Sonoporation System Applications}

This device has the potential to be highly efficient, in terms of both transfection effectiveness and throughput capabilities. It has already been tested on a variety of cell types including cancer cells, red blood cells, and immune cells. This system shows 
promising applications in blood drying technology, and has recently focused on gene therapy applications, specifically CAR-T therapy.

CAR-T therapy is an immunotherapy treatment in which the T-cells of a cancer patient are collected and genetically modified to express the chimeric antigen receptor (CAR) [4]. This therapy programs these immune cells to attack specific tumor cells and eradicate the cancer in a targeted approach. This method has been approved to treat Acute Lymphoblastic Leukemia (ALL) and other types of B cell lymphomas [5]. Viral transfection methods have been shown to be fairly effective for this type of gene therapy, but the manufacturing process for this technique is slow and highly regulated for quality control purposes [4]. The entire process of blood extraction, apheresis for T-cell isolation, T-cell activation, transduction, proliferation, and packaging for transport can take several weeks $[4,5]$. Additionally, the viral transduction method used for this therapy can result in random insertion of the CAR-expressing gene into the T-cell genome [5]. Random insertion could result in toxic immune responses within the patient including cytokine release syndrome and immune effector cell-associated neurotoxicity syndrome [6]. These limitations demonstrate the need for a gene therapy technique that can load T-cells more directly and consistently while cutting down on manufacturing time. With the proper optimization, the microfluidic sonoporation system could be used as an alternative method of T-cell transfection to address these issues.

\section{Problem}

The limitations of both viral and other non-viral transfection techniques demonstrate the need to optimize the microfluidic sonoporation system. Although this 
system has been used experimentally to test the transfection efficiency of various cell types, the optimal parameters for specific cell types and specific applications has yet to be finalized. In order to accomplish this, a characterization of the ultrasound pressures within the microfluidic channels of the device under different driving conditions and chip material/geometric properties is necessary.

To understand how the device performs under different conformations could be accomplished by conducting numerous experimental trials. However, this is a costly and inefficient approach. A better tactic would be to attempt to create a mathematical model characterizing the behavior of the system under different conditions. If the mathematical simulation is robust and accounts for the nuances of the system, it could potentially be used to predict the outcomes of numerous experimental trials almost instantaneously.

\section{Modeling Background}

The basis for the mathematical model developed in this thesis comes from the paper "Modeling of Layered Resonators for Ultrasonic Separation" by Hill et. al. This

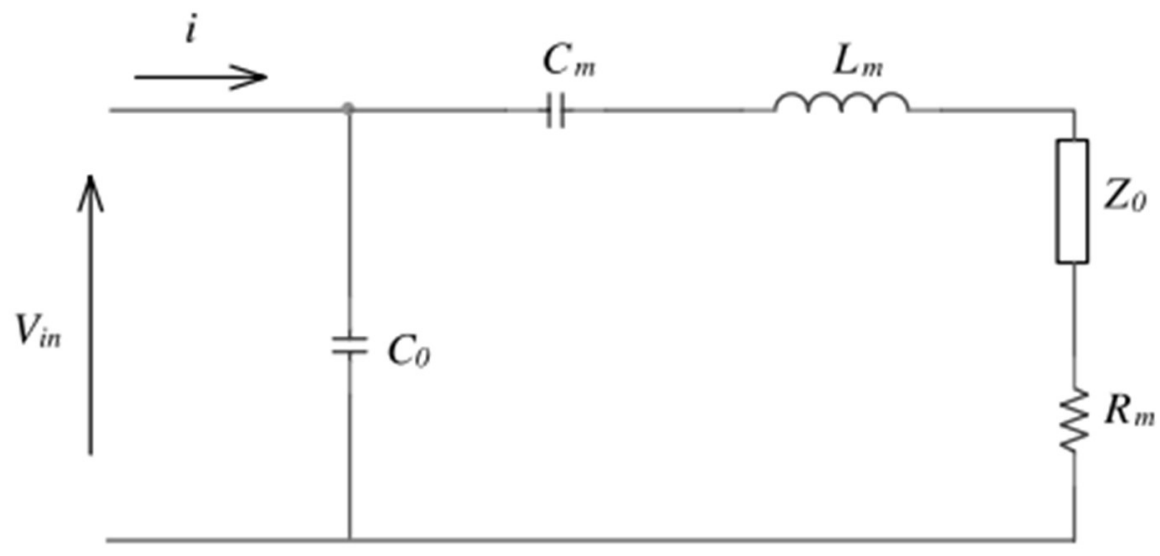

Figure 3: Equivalent-circuit for representing the transducer as a resonance circuit, image from Hill et. 
paper uses an "equivalent-circuit transducer model... coupled with acoustic impedance transfer relationships" to model the electrical and resonance characteristics of a layered cell used for particle separation [7]. The authors represented the behavior of a piezoelectric transducer by modeling it after a resonance circuit (Figure 4). $C_{0}$ represents the static capacitance of the transducer, $C_{m}, L_{m}$, and $R_{m}$, are the mechanical capacitance, inductance, and resistance of the transducer respectively [7]. The most important parameter in this circuit is the variable $Z_{0}$, which represents the input mechanical impedance to the matching layer (layer in contact with transducer) of the resonator cell. Calculating this variable allows force transfer relationships to be calculated, which leads to the generation of a pressure distribution equation showing the values for pressure across the transverse plane of the cell.

One of the goals of this study was to generate data representing the pressure profile across the cell at different transducer driving frequencies. Additionally, Hill et. al generated data to predict filtration performance by calculating the energy-frequency product across a range of frequencies. The authors also validated this data experimentally to determine the model's accuracy and found it matched well with performance data and electrical characteristics [7]. This data is of interest because when translated to the microfluidic sonoporation system it can predict the positions of pressure nodes within the microfluidic channel. Additionally, the model could predict which frequencies show resonant behavior within the device. 


\section{Research Objective}

While translating the Hill model to the microfluidic sonoporation system is one objective of this thesis, another is determining the influence of standing wave behavior on molecular delivery efficiency. The position and number of pressure nodes could be an influential factor in transfection efficiency, and to date no research exists that investigates this question for a microfluidic sonoporation application. The initial hypothesis for this study is that resonant frequencies will create standing waves within the microchannel and the frequencies with the largest acoustic pressures will correlate with high molecular delivery efficiency. By altering the frequency of the transducer and the impedance characteristics of the layers, the standing wave profile can be adjusted to match the realworld behavior of the microfluidic cell. Investigating the effect of pressure nodes on transfection performance is a major focus of this thesis.

\section{Outline}

This thesis consists of three main parts. Part one is the re-creation and validation of the Hill paper model. Through the use of the MATLAB environment, the relevant equations, mechanical and geometric parameters, and electrical values from the Hill study are replicated. The data and graphs of interest are simulated to determine if the same results from the Hill paper can be produced to validate the model's legitimacy.

Part two is the translation of this model to the microfluidic sonoporation system. This step includes applying the same impedance and force transfer equations that were described in the Hill paper, but with altered electrical characteristics and values for material and geometric parameters to represent the PDMS-based prototype device. The 
same graphs of interest from the Hill paper are generated for a variety of parameters relevant to the prototype design in order to investigate their effect on the system's behavior.

Part three is the experimental comparison of the translated model. Experimental trials testing the molecular delivery efficiency are conducted at specified driving conditions to compare the results to those predicted by the model. Following this is a presentation and discussion of the results from each step. The paper concludes with closing remarks, limitations, and future works outlining where this research fits in the spectrum of literature and what steps should be taken next. 


\section{METHODS}

\section{Hill Paper Validation}

The Hill paper is a relevant model for translation to the microfluidic system because the authors investigate the behavior of a layered resonator in which "a piezoelectric transducer is bonded to a carrier or matching layer which in turn drives a fluid layer...and which is terminated by a reflector layer" [7]. As shown in Figure 3, the microfluidic chip is a layered cell with a PZT transducer bonded to a silicone layer which drives into a

microfluidic channel layer and is terminated by a reflector layer (PDMS). The authors of the Hill paper wish to understand how the material and geometric parameters influence the performance of ultrasonic particle separation, while the investigative goal of this paper is to understand those parameters' influence on the efficiency of molecular delivery. Although the objectives are different, the focus on standing wave behavior within an ultrasonic resonator is the same.

The Hill paper validation required understanding how the equivalent-circuit transducer model was developed. The critical element of the circuit is the component $Z_{0}$ because this represents the mechanical input impedance to the matching layer of the resonator. Calculating the input impedance to the matching layer will allow subsequent equations to be developed which culminate in an equation that describes the spatial 
variation of acoustic pressure across the cell. The spatial variation of acoustic pressure through the microchannel is the most important equation because it predicts the positions at which there are positive and negative pressure nodes. Nodal positioning will influence particle location and is expected to have an impact on the cell's performance. Developing the acoustic pressure equation requires an understanding of how the preceding equations were developed which follows here. All equations are given by Kinsler et. al [9] as referenced in the Hill study.

\section{Mechanical Input Impedance:}

$$
Z_{0}=r_{m} S \frac{Z_{f}+j r_{m} S \tan k_{m} t_{m}}{r_{m} S+j Z_{f} \tan k_{m} t_{m}}
$$

This equation represents the input mechanical impedance to the matching layer. The term $r_{m}$ is the specific acoustic impedance of the matching layer, $\mathrm{S}$ is the crosssectional area of the cavity, $Z_{f}$ is the mechanical impedance looking into the layer adjacent to the matching layer, $k_{m}$ is the wavenumber in the matching layer, and $t_{m}$ is the thickness of the matching layer. This is a complex number as shown by the element $j$, to allow for losses through the layer. If the material and geometric properties of the matching layer are known, then every variable in this equation can be determined aside from $Z_{f}$. To calculate $Z_{f}$, an impedance transfer relationship is used in which $Z_{f}$ is the variable on the left-hand side of eq. (1) and the layer adjacent to that layer would be the new unknown impedance value. This transfer relationship would continue until an acoustic free-field boundary is reached (region where no reflections occur). This field, typically air, has a mechanical input impedance equal to its specific acoustic impedance 
which can be calculated from the material properties of air. Once this quantity is known, the mechanical impedance values can be worked through backwards starting with the terminating layer adjacent to the free field until the matching layer is reached. This process is shown in the diagram below for a representative cell (Figure 5).

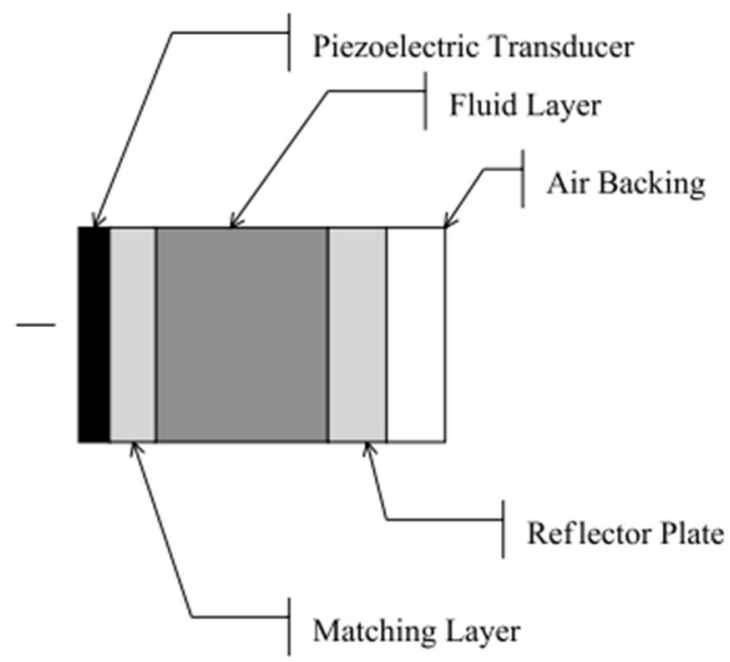

Figure 5a: Schematic diagram of basic resonator cell, image from Hill et. al

$$
\begin{gathered}
Z_{\text {fluid }}=r_{f} S \frac{Z_{\text {ref.plate }}+j r_{f} S \tan k_{f} t_{f}}{r_{f} S+j Z_{\text {ref } \text { plate }} \tan k_{f} t_{f}} \Rightarrow Z_{\text {ref.plate }}=r_{r} S \frac{Z_{\text {air }}+j r_{r} S \tan k_{r} t_{r}}{r_{r} S+j Z_{\text {air }} \tan k_{r} t_{r}} \\
\Rightarrow Z_{\text {air }}=r_{\text {air }}=\text { Acoustic Impedance }=\rho_{\text {air }} * C_{\text {air }}
\end{gathered}
$$

Figure 5b: Impedance transfer relationship process shown for figure 5a example

The acoustic impedance equation equates to the density of the material $\left(\rho_{\text {air }}\right)$ times the speed of sound through that material $\left(c_{\text {air }}\right)$. This value can be plugged into the equation for $Z_{\text {ref.plate, }}$ and that solution can be plugged into the equation for $Z_{\text {fluid }}$ whose 
result will allow for the calculation of the input mechanical impedance into the matching layer. Once this solution is determined, the force generated by the transducer acting on the matching layer can be calculated.

Force generated by transducer:

$$
F_{0}=\frac{\varphi V Z_{0}}{Z_{e t}+Z_{0}}
$$

In this equation, $\varphi$ is the transformation ratio between mechanical and electrical quantities, $\mathrm{V}$ is the input voltage to the transducer and $Z_{e t}$ is the mechanical impedance of the transducer at the output terminals when they are short-circuited. This force in turn generates a force between the matching layer and the layer next to it. In the schematic from the Hill paper, this would be the fluid layer, $F_{f}$.

Force acting on layer adjacent to matching layer:

$$
F_{f}=\frac{F_{0} Z_{f}}{Z_{f} \cos k_{m} t_{m}+j r_{m} S \sin k_{m} t_{m}}
$$

The variables in this equation represent the same quantities as in previous equations where the subscript $f$ is for the fluid layer and the subscript $m$ is for the matching layer. If there are multiple layers between the transducer and the fluid layer, as is the case for the Hill study's experimental cell, the force transfer relationship can be used on successive layers to calculate the force at the fluid boundary.

Once the force at the fluid boundary is determined, then the spatial variation of the acoustic pressure through the fluid layer can be calculated. If $x=0$ at the boundary 
between the layer adjacent to the fluid layer and the fluid layer, then the pressure can be expressed as follows.

Spatial variation of acoustic pressure:

$$
p(x)=\frac{F_{f}}{S} \frac{Z_{r} \cos k_{f}\left(t_{f}-x\right)+j r_{f} S \sin k_{f}\left(t_{f}-x\right)}{Z_{r} \cos k_{f} t_{f}+j r_{f} S \sin k_{f} t_{f}}
$$

In eq. (4), $Z_{r}$ is the mechanical impedance looking into the reflector layer which follows the fluid layer. The subscripts and variable representations are the same as in the other equations.

Additional equations applied in the development of this model include the equations for calculating angular frequency (eq. 5), wavenumber (eq. 6), and acoustic impedance (eq. 7).

$$
\begin{aligned}
& \omega=2 * \pi * f \\
& \lambda=\omega / C \\
& r=\rho * C
\end{aligned}
$$

In the equations above, $f$ represents the driving frequency of the transducer, $\omega$ is the angular frequency, $c$ is the acoustic velocity of the material, and $\rho$ is the density of the material.

The authors of Hill et. al applied these equations to their experimental layered resonator, taken from Hawkes and Coakley [10], shown in (Figure 6). 


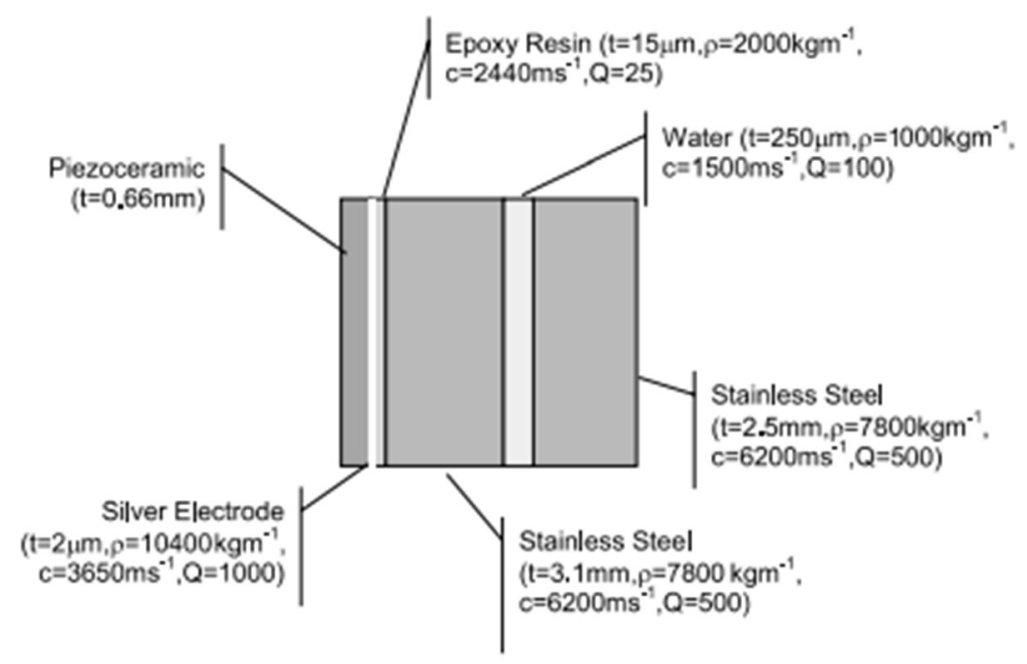

Figure 6: Schematic of Hawkes and Coakley ultrasonic resonator, image from Hill et. al

Validating the Hill paper began with determining the value of eq. (1) and working towards eq. (4) by applying the transfer relationships to the cell in Figure 6. The steps to reach that point are described as follows:

1. Determine acoustic impedance of air backing (free-field boundary)

2. Calculate mechanical impedance (eq. 1) of stainless-steel reflector layer using value from step 1

3. Calculate mechanical impedance of fluid layer using value from step 2

4. Calculate mechanical impedance of initial stainless-steel layer using value from step 3

5. Calculate mechanical impedance of epoxy resin layer using value from step 4

6. Calculate mechanical impedance of silver electrode (matching layer) using value from step 5

7. Calculate force generated by transducer (eq. 2) acting on matching layer using value from step 6 and estimates of other quantities 
8. Calculate force acting on epoxy resin layer (eq. 3) using value from step 7

9. Calculate force acting on first stainless-steel layer with transfer relationship (eq.

3) using value from step 8

10. Calculate force acting on the fluid layer at the stainless-steel/fluid layer boundary using value from step 9

11. Determine the spatial variation of acoustic pressure with eq. (4) using value from step 10

The known material and geometric parameters are presented in the table below (Table 1).

Table 1: Material and geometric parameters for Hill layered cell

\begin{tabular}{|c|c|c|c|c|c|}
\hline Parameters & $\begin{array}{l}\text { Thicknes } \\
\text { s (t) } \\
(\mathrm{mm})\end{array}$ & $\begin{array}{l}\text { Densit } \\
y(\rho) \\
\left(\mathrm{kg} / \mathrm{m}^{3}\right. \\
)\end{array}$ & $\begin{array}{c}\text { Acousti } \\
\text { c } \\
\text { Velocit } \\
\text { y (c) } \\
(\mathrm{m} / \mathrm{s}) \\
\end{array}$ & $\begin{array}{l}\text { Wavenumbe } \\
r(\mathbf{k})(\mathrm{rad} / \mathrm{m})\end{array}$ & $\begin{array}{l}\text { Acoustic Impedance } \\
(\mathrm{r})\left(\mathrm{kg} / \mathrm{s}^{*} \mathrm{~m}^{2}\right)\end{array}$ \\
\hline Air & $\infty$ & 1.204 & 343 & N/A & $\mathrm{C}_{\mathrm{air}}{ }^{*} \rho_{\mathrm{air}}=412.972$ \\
\hline $\begin{array}{c}\text { Stainless Steel } \\
\text { Backing }\end{array}$ & 2.5 & 7800 & 6200 & $\omega / C_{S S B}$ & $C_{S S B}{ }^{*} \rho_{S S B}=48,360,000$ \\
\hline $\begin{array}{c}\text { Fluid Channel } \\
\text { (Water) }\end{array}$ & 0.25 & 1000 & 1500 & $\omega / C_{\text {Water }}$ & $\begin{array}{c}C_{\text {Water }}{ }^{*} \rho_{\text {Water }}= \\
1,500,000\end{array}$ \\
\hline Stainless Steel Initial & 3.1 & 7800 & 6200 & $\omega / C_{S S I}$ & $C_{S S I} * \rho_{S S I}=48,360,000$ \\
\hline Epoxy Resin & 0.015 & 2000 & 2440 & $\omega / C_{E R}$ & $C_{E R} * \rho_{E R}=4,880,000$ \\
\hline Silver Electrode & 0.002 & 10,400 & 3650 & $\omega / C_{S E}$ & $C_{S E}{ }^{*} \rho_{S E}=37,960,000$ \\
\hline
\end{tabular}


Additional values used to calculate the force generated by the transducer and the wavenumber are presented in Table 2.

Table 2:Additional values for developing resonator model

\begin{tabular}{|c|c|c|c|c|}
\hline $\begin{array}{l}\text { Cross-sectional } \\
\text { Area of Fluid } \\
\text { Channel }\left(\mathbf{m}^{2}\right)\end{array}$ & $\begin{array}{l}\text { Transducer } \\
\text { Output } \\
\text { Voltage (V) }\end{array}$ & $\begin{array}{l}\text { Frequency } \\
\text { (MHz) }\end{array}$ & $\begin{array}{c}\text { Transformation } \\
\text { Ratio ( } \phi)\end{array}$ & $\begin{array}{c}\text { Transducer } \\
\text { Shorted } \\
\text { Terminal } \\
\text { Impedance }\left(\mathrm{Z}_{\mathrm{ET}}\right)\end{array}$ \\
\hline $\begin{array}{c}0.01 * 0.00025= \\
2.5 \times 10^{-6}\end{array}$ & $\begin{array}{c}V(f)=[1.05, \\
1.6,1.075, \\
1.95]\end{array}$ & $\begin{array}{c}f=[2.75, \\
2.92,3.00, \\
3.09]\end{array}$ & Variable [0.1 -1] & $\sim 1$ \\
\hline
\end{tabular}

The cross-sectional area of the fluid channel is given by Hawkes and Coakley [10] who originally developed the layered chip. The transformation ratio is a unitless transducer specific value and dependent on the input frequency to the transducer, but it is not provided by Hill et. al. During validation, this value is originally approximated as 1 then altered to scale the resultant data to match that found by Hill et. al. As this value is frequency dependent, the transformation ratio was altered slightly for each driving frequency to better match the data. The mechanical impedance across the shorted transducer terminals is unknown and estimated as 1 throughout validation. The values for voltage correspond to the values for frequency, i.e. $V=1.05$ volts corresponds to $f=2.75$ $\mathrm{MHz}$ and so on. These values come from a figure (Figure 7) in Hill et. al that shows modeled and measured voltages across the transducer across a range of frequencies. 


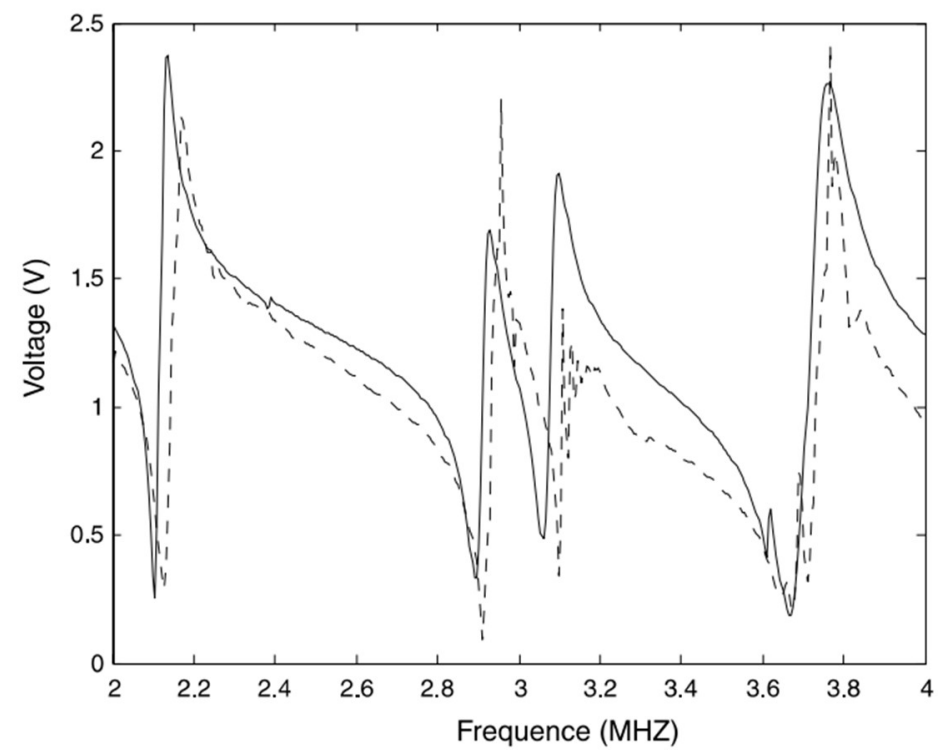

Figure 7: Voltage across the transducer terminals for the layered resonator filled with water, image from Hill et. al

The frequencies at which the voltage values are determined are based on another figure (Figure 8) from Hill et. al which shows the modelled energy-frequency product and measured clearance across a range of frequencies. These terms measure the performance of the cell and for the authors this was a measure of filtration/separation efficiency (clearance). 


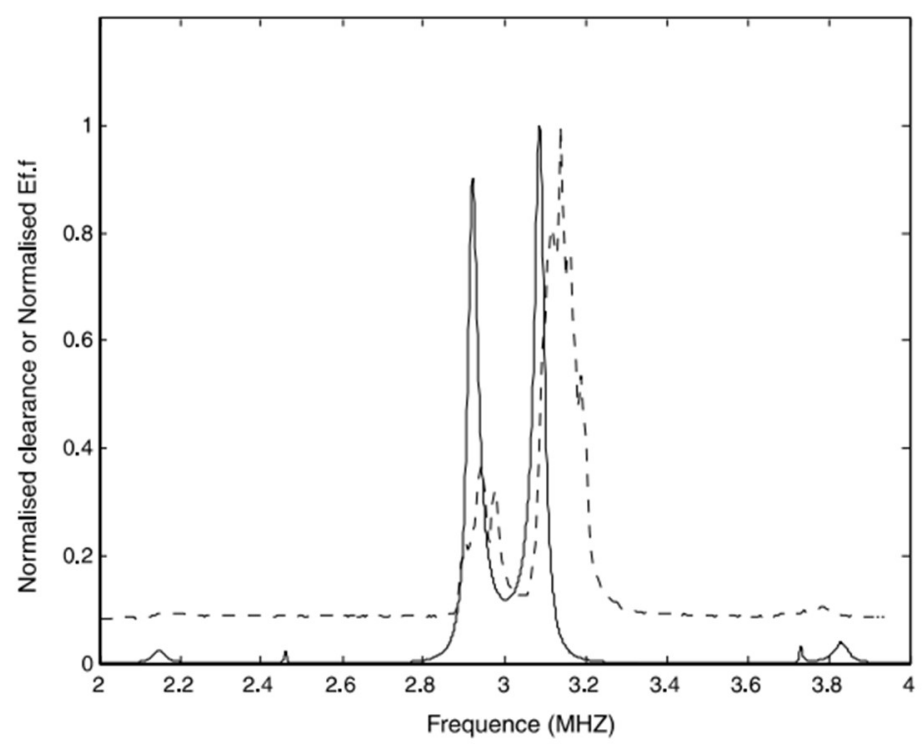

Figure 8: Normalized clearance or Ef*f product for the resonator, image from Hill et. al

Figure 8 shows two peaks where the clearance is most efficient, one at $2.92 \mathrm{MHz}$ and the other at 3.09 MHz. The authors used these two frequencies as well as the frequency at the dip between peaks, $3.0 \mathrm{MHz}$, and a random frequency of $2.75 \mathrm{MHz}$ to model the acoustic pressure profile across the cell as shown in Figure 9. The method used to determine the voltages corresponding to these select frequencies from Figure 7 is shown in Figure 10. 


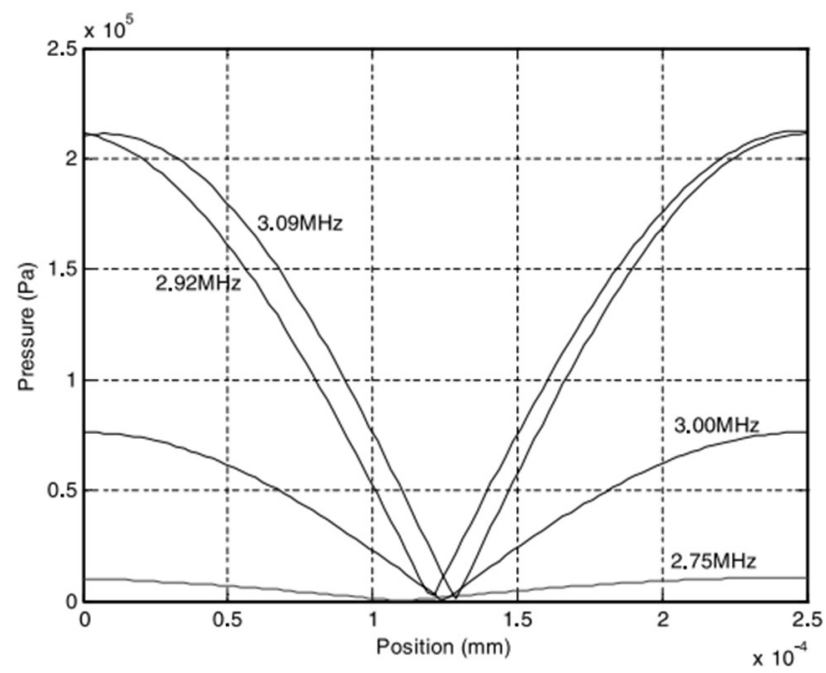

Figure 9: Acoustic pressure profile across the cell at peak clearance frequencies and 2, image from Hill et. al

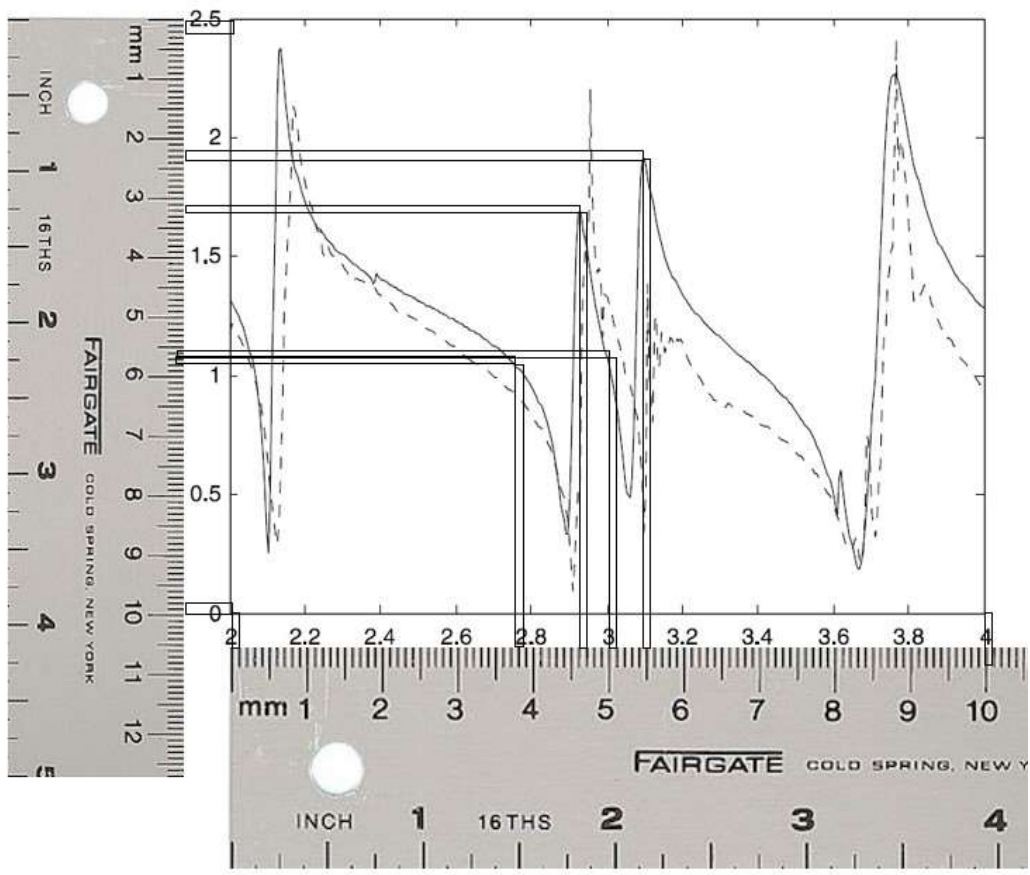

Figure 10: Method for determining voltages corresponding to select frequencies. Rulers were used as a representative scale on each axis to determine the quantities between tick marks.

Figures 8 and 9 are the relevant graphs for replication from this model. The acoustic pressure profile in Figure 9 can be applied to the microfluidic device to determine 
the location of standing waves and the pressure magnitudes expected at these locations for specific frequencies. Additionally, Figure 8 can be replicated in the microfluidic device by calculating the maximum pressure across a range of frequencies from eq. (4) and plotting those values against their corresponding frequencies.

After determining all relevant quantities, steps 1-11 are programmed within MATLAB to generate an acoustic pressure profile across the cell at the select frequencies. The normalized clearance graph is replicated by running the acoustic pressure equation across a range of frequencies. The graphs developed within MATLAB are compared to Figures 8 and 9 to determine the validity of the Hill model. These results are presented in section III.

\section{Microfluidic Sonoporation System Translation}

After the relevant data from the Hill study is validated, the same modelling process is applied to the microfluidic sonoporation system. Translating the same equations and transfer relationships to the microfluidic device requires information about the material and geometric properties of the layered chip, as well as information about the transducer voltage output at specific frequencies. Figure 3 shows the order of layers for the sonoporation resonator cell; in this figure the silicone layer is the matching layer, adjacent to that is a glass slide layer which forms a boundary with the microfluidic channel, and the terminating/reflection layer is composed of PDMS. Geometric parameters for these layers were determined by measurement or knowledge of chip dimensions. Densities are sourced from a material database referenced later. Acoustic 
velocities are taken from additional sources and scientific literature that conducted studies on the acoustic velocity of the materials used in the microfluidic chip.

The constitution of the fluid layer is complex as it composed of a buffered saline solution that contains microbubbles and a dilute concentration of cells. The properties of this layer are estimated as both water and whole blood for purposes of simplifying the calculations. Table 3 shows the geometric and material parameters for the microfluidic chip.

Table 3: Material and geometric parameters for the microfluidic device. Fluid channel properties are estimated as both water and whole blood.

\begin{tabular}{|c|c|c|c|c|c|}
\hline $\begin{array}{l}\text { Pardyeters } \\
\text { Layers }\end{array}$ & $\begin{array}{l}\text { Thickness } \\
\text { (t) (mm) }\end{array}$ & $\begin{array}{l}\text { Density } \\
\qquad(\rho) \\
\left(\mathrm{kg} / \mathrm{m}^{3}\right)\end{array}$ & $\begin{array}{l}\text { Acoustic } \\
\text { Velocity } \\
\text { (c) }(\mathrm{m} / \mathrm{s})\end{array}$ & $\begin{array}{c}\text { Wavenumber } \\
\text { (k) (rad/m) }\end{array}$ & $\begin{array}{c}\text { Acoustic } \\
\text { Impedance }(\mathrm{r}) \\
\left(\mathrm{kg} / \mathrm{s}^{*} \mathrm{~m}^{2}\right)\left(\times 10^{5}\right)\end{array}$ \\
\hline Silicone & 1.0 & $\begin{array}{l}700- \\
3800 \\
\text { Avg: } \\
1250\end{array}$ & $\begin{array}{l}960- \\
1110 \\
\text { Avg: } \\
1035\end{array}$ & $\omega_{\mathrm{si}} / c_{\mathrm{Si}}$ & $\begin{array}{c}\rho_{\mathrm{Si}_{\mathrm{i}}}{ }^{*} \mathrm{C}_{\mathrm{Si}}=[7.245 ; \\
12.9375 ; 39.33 \\
\quad 12 ; 13.875]\end{array}$ \\
\hline $\begin{array}{c}\text { Borosilicate } \\
\text { Glass }\end{array}$ & 1.0 & 2400 & 5640 & $\omega_{B G} / c_{B G}$ & $\rho_{B G} * C_{B G}=135.36$ \\
\hline $\begin{array}{l}\text { Microchannel } \\
\text { Fluid: Water }\end{array}$ & 0.2 & 1000 & 1481 & $\omega_{\text {water }} / c_{\text {water }}$ & $\rho_{\text {water }}{ }^{*} C_{\text {water }}=14.81$ \\
\hline $\begin{array}{c}\text { Microchannel } \\
\text { Fluid: Whole } \\
\text { Blood }\end{array}$ & 0.2 & 1060 & 1578 & $\omega_{\text {blood }} / c_{\text {blood }}$ & $\begin{array}{c}\rho_{\text {blood }}{ }^{*} C_{\text {blood }}= \\
16.7268\end{array}$ \\
\hline PDMS & 4.0 & 965 & $\begin{array}{c}1076.5- \\
1250 \\
\text { Avg: } \\
1119\end{array}$ & $\omega_{\text {PDMS }} / C_{\text {PDMS }}$ & $\begin{array}{c}\text { PPDMS }{ }^{*} \text { CPDMS }=[\min : \\
\text { 10.38822; avg: } \\
\text { 10.79835; max: } \\
12.0625]\end{array}$ \\
\hline
\end{tabular}

As shown in Table 3, both silicone and PDMS exhibit variability in their acoustic impedance values. This arises from the range of acoustic velocities reported for each material and the range of densities reported for silicone. The values for silicone acoustic 
impedance are calculated by taking the minimum, average, and maximum reported values for density and multiplying by the average value for acoustic velocity. The same thing is done with the three values for silicone acoustic velocity by multiplying by the average silicone density to attain the other impedance values. The average value for silicone density comes from the averaged densities over a dataset of silicone materials. The average value for acoustic velocity through silicone is calculated by averaging the minimum and maximum values. The average value for acoustic velocity through PDMS is taken as the value reported most consistently throughout the literature. A similar approach was taken for the PDMS impedance values but with a constant density value. This table shows large variability in the possible properties for the chip layers so an investigation of the impact of changing these parameters is conducted.

The output voltage of the transducer at specific driving frequencies is determined by a calibration of the transducer using a hydrophone-water tank setup. A hydrophone measures acoustic wave pressure and converts it to a voltage. The experimental setup (Figure 11) connected a function generator to the transducer which was suspended in a tank of water. The hydrophone was placed several centimeters away from the transducer; this distance was held constant throughout the calibration. The hydrophone was connected to the oscilloscope which displayed the voltage waveform and quantities. 


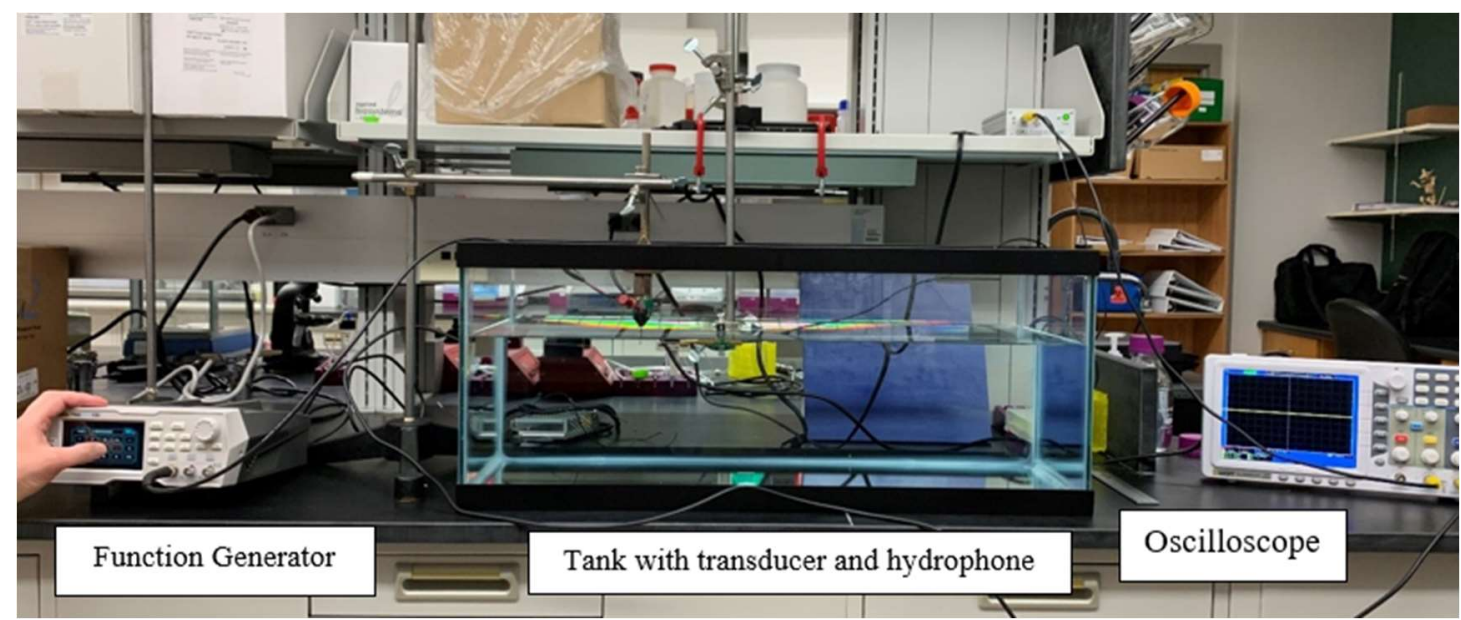

Figure 11: Transducer hydrophone calibration setup

A test was conducted to determine the output voltage of the transducer across a range of driving frequencies. An applied 20 volts was maintained while the frequency was altered by increments of $0.5 \mathrm{MHz}$ beginning at $3 \mathrm{MHz}$ and ending at $8 \mathrm{MHz}$. The output of the hydrophone is presented in Figure 12.

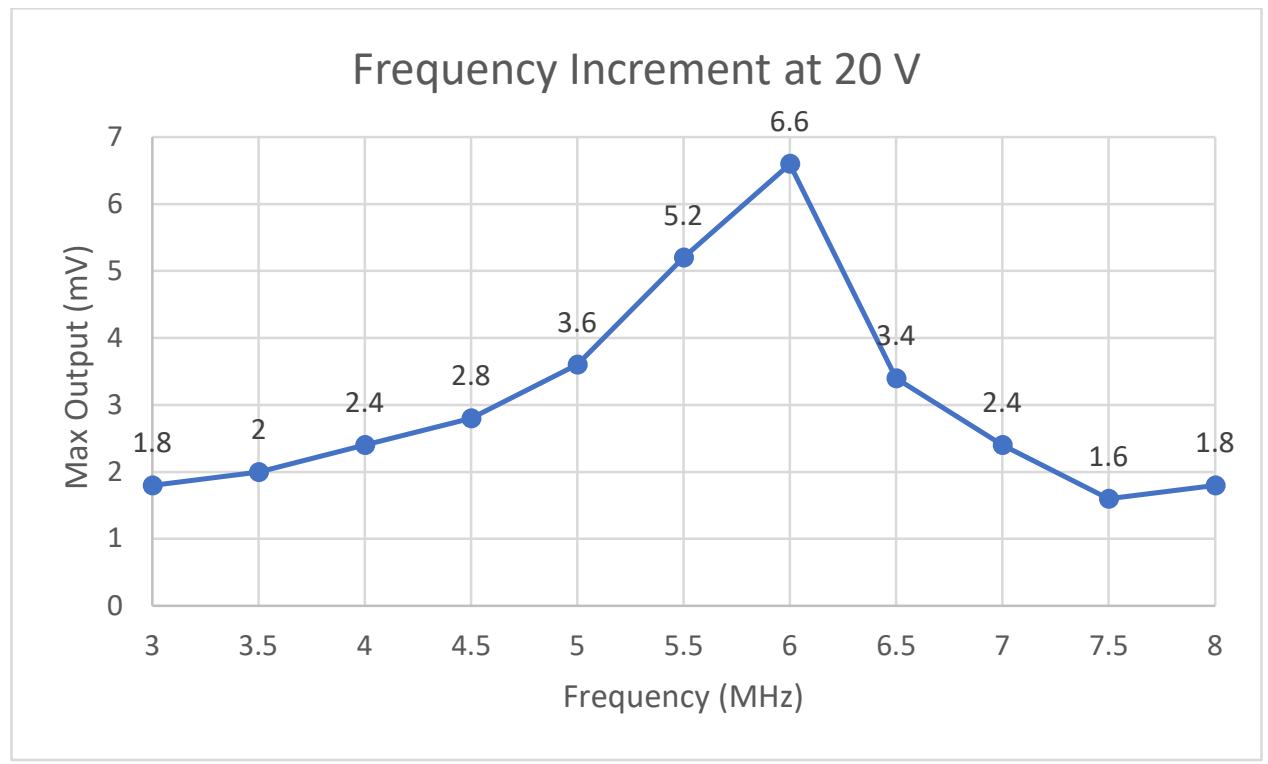

Figure 12: Hydrophone output (transducer output) across a range of frequencies 
Additional values used to calculate eq. (2) for the microfluidic device and the wavenumber are shown in Table 4.

Table 4: Electrical values for the PZT transducer and the driving frequencies as well as fluid channel cross sectional area.

\begin{tabular}{|c|c|c|c|c|}
\hline $\begin{array}{l}\text { Cross-Sectional } \\
\text { Area of Fluid } \\
\text { Channel }\left(\mathbf{m}^{2}\right)\end{array}$ & $\begin{array}{c}\text { Transducer } \\
\text { Output Voltage } \\
\text { (V) }\end{array}$ & $\begin{array}{l}\text { Frequency } \\
\text { (MHz) }\end{array}$ & $\begin{array}{c}\text { Transformation } \\
\text { Ratio }(\phi)\end{array}$ & $\begin{array}{c}\text { Transducer } \\
\text { Shorted Terminal } \\
\text { Impedance }\left(\mathrm{Z}_{\mathrm{ET}}\right)\end{array}$ \\
\hline $\begin{array}{c}\left(200 \times 10^{-6}\right)^{2}= \\
4 \times 10^{-8}\end{array}$ & $\begin{array}{c}V(f)=\left[2.4 \times 10^{-3}\right. \\
6.6 \times 10^{-3} ; \\
\left.1.8 \times 10^{-3}\right]\end{array}$ & $f=[4 ; 6 ; 8]$ & 1 & 1 \\
\hline
\end{tabular}

The values for transformation ratio and the terminal impedance of the transducer when shorted are unknown. Unlike in the Hill validation, the transformation ratio cannot be scaled for adjustment because there are no results to match the new data to. The values for frequency are chosen because they match the driving frequency of the transducer in experimental trials of calcein delivery.

The same steps that were taken in the Hill validation for developing equations 1-4 are applied to the microfluidic device. The microfluidic chip is surrounded by a field of air, so the acoustic impedance of air is used to work backwards through the layers beginning with PDMS and ending with silicone to determine the input mechanical impedance of the silicone (matching) layer. This input mechanical impedance is applied to eq. (2) to determine the transducer force driving into the matching layer. The force transfer relationship from eq. (3) is applied to the glass slide layer and subsequently the 
fluid layer to determine the force driving into the fluid layer. This fluid force is input into eq. (4) to determine the acoustic pressure profile across the microfluidic channel.

As mentioned earlier, the variation in reported values for some of the layers prompted an investigation into the impact of these differences. This investigation is conducted by creating a control of all variables except one, using their average, modal, or singularly reported values, and altering the other variable to understand how it impacts the pressure profile across the cell. An investigation of the impact of the fluid layer properties is also included by running the model at water values and then at whole blood values. The pressure profile for each iteration of the study was analyzed at 4,6 , and 8 $\mathrm{MHz}$ with the corresponding output voltage for each frequency.

The first variable studied was the density of silicone. The model was evaluated at the minimum, average, and maximum values for silicone density, at 4, 6, and $8 \mathrm{MHz}$, for both water and blood fluid layers. All other values were held at their average or singularly reported values.

The second variable studied was the acoustic velocity of silicone. The model was evaluated at the minimum, average, and maximum values for silicone acoustic velocity, at 4, 6, and $8 \mathrm{MHz}$, for both water and blood fluid layers. All other values were held at their average or singularly reported values.

The third variable studied was the acoustic velocity of PDMS. The model was evaluated at the minimum, average, and maximum values for PDMS acoustic velocity, at 4, 6, and $8 \mathrm{MHz}$, for both water and blood fluid layers. All other values were held at their average or singularly reported values. 
The fourth variable studied was the impact on thickness alterations. Although the layer thicknesses are constant right now, a variation in layer thicknesses was expected in future iterations of this design and is therefore important to investigate. The silicone, glass, and PDMS layers were scaled to both half and double thickness independently as the microfluidic channel thickness was held constant to show how the position of pressure nodes changes. All other variables were held at their averages.

After developing graphs showing the pressure profile across the cell for different parameters, Figure 8 (normalized clearance) was replicated by evaluating the model at the frequency steps and corresponding voltages from Figure 12. At each step, the pressure equation was calculated and the maximum pressure value across the cell was recorded. Voltages are interpolated between 3 and $8 \mathrm{MHz}$ at 450 times the sampling rate of the original data set from calibration. Material and geometric parameters were taken as their average values. After iterating through each step, a graph displaying the maximum pressures vs. their corresponding frequency was created which shows the frequencies at which the model predicts resonant behavior.

\section{Experimental Trials}

To understand how this model relates to the experimental results, an experiment was conducted to measure fluorescence intensity within red blood cells (RBCs). This experiment uses calcein as the molecular component for delivery and flow cytometry to measure cellular uptake of the compound. The steps for conducting this experiment at different ultrasonic frequencies is shown below.

1. Keep all samples at room temperature during experiment 
2. Dilute human RBCs in $17 \mathrm{~mL}$ PBS to a final concentration of 50 million $/ \mathrm{mL}$

1. Aliquot $3 \mathrm{~mL}$ for no calcein control group (group "a")

2. Aliquot $13.5 \mathrm{~mL}$ for calcein-groups:

a. Add $1.5 \mathrm{~mL}$ of calcein (from $1 \mathrm{mg} / \mathrm{mL}$ calcein stock solution) to the $13.5 \mathrm{~mL}$ aliquot

3. Divide calcein groups into 3-mL aliquots

4. Experiment groups ( $\mathrm{n}=3$ /group, you can run $3 \mathrm{~mL}$ through device using $50-\mathrm{mL}$ conical vials then divide into 1-mL samples for measurements):

1. No calcein control (no flow, no ultrasound)

2. No treatment (calcein added but no flow, no ultrasound)

3. Flow through spiral-channel microfluidic device using the $600 \mathrm{~mL} / \mathrm{h}$ setting for an empty $60 \mathrm{~mL}$ syringe $(27.6 \mathrm{~mm}$ diameter), without ultrasound (with calcein added)

4. Flow through spiral-channel microfluidic device using the $600 \mathrm{~mL} / \mathrm{h}$ setting for an empty $60 \mathrm{~mL}$ syringe (27.6mm diameter), with $25 \mu \mathrm{L} / \mathrm{mL}$ of cationic microbubbles $+4 \mathrm{MHz}$ ultrasound at 20 setting on function generator (with calcein added)

5. Flow through spiral-channel microfluidic device using the $600 \mathrm{~mL} / \mathrm{h}$ setting for an empty $60 \mathrm{~mL}$ syringe $(27.6 \mathrm{~mm}$ diameter), with $25 \mu \mathrm{L} / \mathrm{mL}$ of cationic microbubbles $+6 \mathrm{MHz}$ ultrasound at $20 \mathrm{~V}$ setting on function generator (with calcein added)

6. Flow through spiral-channel microfluidic device using the $600 \mathrm{~mL} / \mathrm{h}$ setting for an empty $60 \mathrm{~mL}$ syringe $(27.6 \mathrm{~mm}$ diameter), with $25 \mu \mathrm{L} / \mathrm{mL}$ 
of cationic microbubbles $+8 \mathrm{MHz}$ ultrasound at $20 \mathrm{~V}$ setting on function generator (with calcein added)

5. After treatment wash all samples $3 x$ (including control groups)

a. Centrifuge with program \#3 (1500g for 5min)

b. Aspirate supernatant, resuspend in $1 \mathrm{~mL}$ PBS

c. Centrifuge with program $\# 3$ (1500g for $5 \mathrm{~min})$

d. Aspirate supernatant, resuspend in $1 \mathrm{~mL}$ PBS

e. Centrifuge again with program \#3 (1500g for $5 \mathrm{~min})$

f. Aspirate supernatant, resuspend in $250 \mu \mathrm{L}$ PBS

6. Run flow cytometry on samples

A chart was generated which shows the relative fluorescence intensity within the RBCs for no calcein and no ultrasound, calcein with no ultrasound and no flow, calcein with flow and no ultrasound, and calcein with flow and ultrasound at 4, 6, and $8 \mathrm{MHz}$. ANOVA tests to determine statistical significance were conducted between these groups. 


\section{RESULTS AND DISCUSSION}

\section{Hill Paper Validation}

The first portion of the Hill study validation was replicating Figure 9. Shown in

Figure 13 is the acoustic pressure profile across the cell with a transformation ratio set to 1.

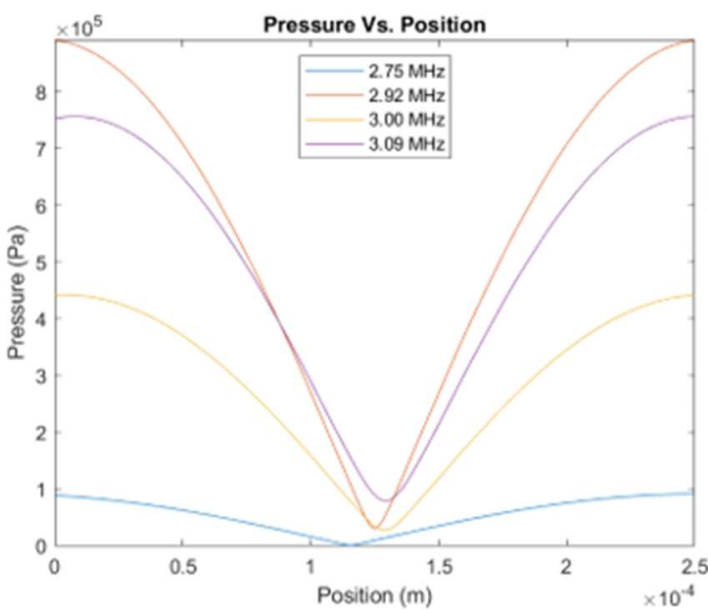

(a)

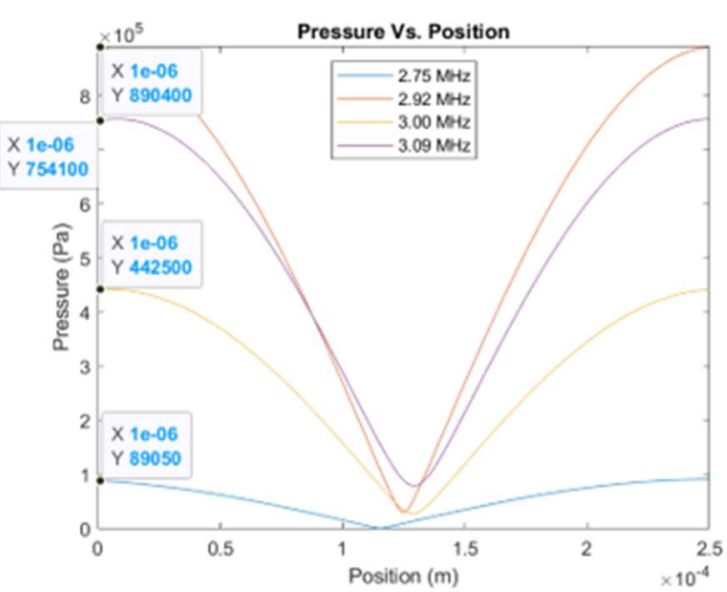

(b)

Figure 13: Acoustic pressure profile across cell with non-specific transformation ratios and left boundary values shown. Left boundaries used to scale for specific transformation ratios.

The values on the left boundary (y-axis) of the cell were compared to the values on the left boundary for Figure 9 and ratios were developed to produce more specific 
transformation ratios (scaling factors) for each frequency. Table 5 shows the transformation ratio used for each frequency.

Table 5: Specific transformation ratios for each driving frequency from Figure 13. Values to calculate transformation ratio taken from Figure $13 \mathrm{~b}$ and left boundary values of Figure 9.

\begin{tabular}{c|c|c|c|c}
$\begin{array}{c}\text { Frequency } \\
\text { (MHz) }\end{array}$ & 2.75 & 2.92 & 3.00 & 3.09 \\
\hline $\begin{array}{c}\text { Transformation } \\
\text { Ratio }\end{array}$ & $\begin{array}{c}0.1 / 0.8905= \\
0.112\end{array}$ & $\begin{array}{c}2.1 / 8.904= \\
0.236\end{array}$ & $\begin{array}{c}0.75 / 4.423= \\
0.17\end{array}$ & $\begin{array}{c}2.1 / 7.532= \\
0.28\end{array}$
\end{tabular}

The same acoustic profile was then generated using the specific transformation ratios from Table 5. This graph, as well as a comparison to Figure 9, is shown below in Figure 14.
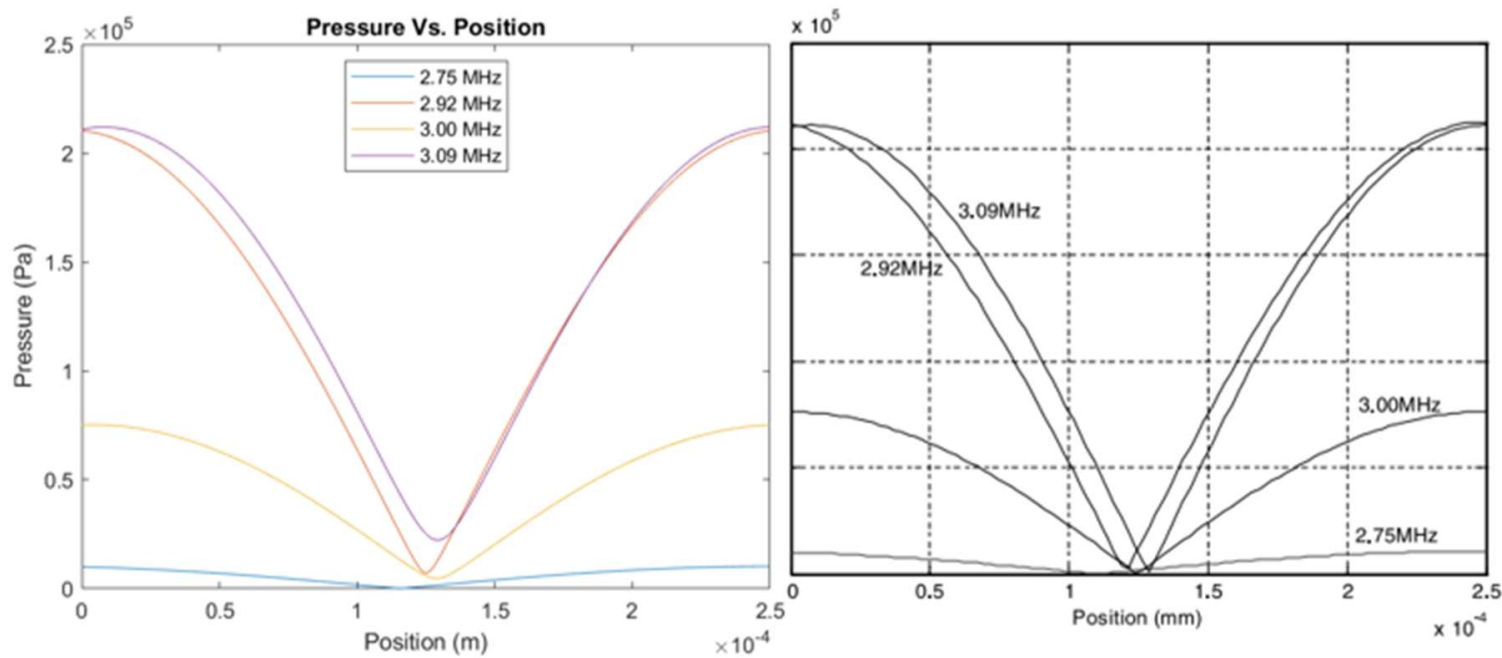

Figure 14: (Left) Graph generated by replicated model using specific transformation ratios (Right) Figure 9 from Hill being replicated.

Figure 14 shows a good match between the replicated model on the left and the graph from Hill et. al on the right. The magnitudes of the pressures match well on the boundaries, the shapes of the curves match well, and the position of the low-pressure 
nodes match well also. The low-pressure node for $3.09 \mathrm{MHz}$ lies to the right of the middle of the cell in both graphs, for $2.92 \mathrm{MHz}$ and $2.75 \mathrm{MHz}$ it lies to the left in both graphs, and for $3.00 \mathrm{MHz}$ it lies in the center for both graphs.

The next step of the Hill study validation was replicating Figure 8. The maximum pressure across the cell was found across a range of frequencies from 2-4 MHz stepping up by $1000 \mathrm{~Hz}$ each time. The voltages for 21 points on Figure 7 starting with $2 \mathrm{MHz}$ and stepping up by $0.1 \mathrm{MHz}$ were determined with the same method shown in Figure 10. Voltages for frequencies between these steps were found by interpolation at a sampling rate that matched a frequency step of $1000 \mathrm{~Hz}$. Figure 15 shows the maximum pressure across the cell using this method.

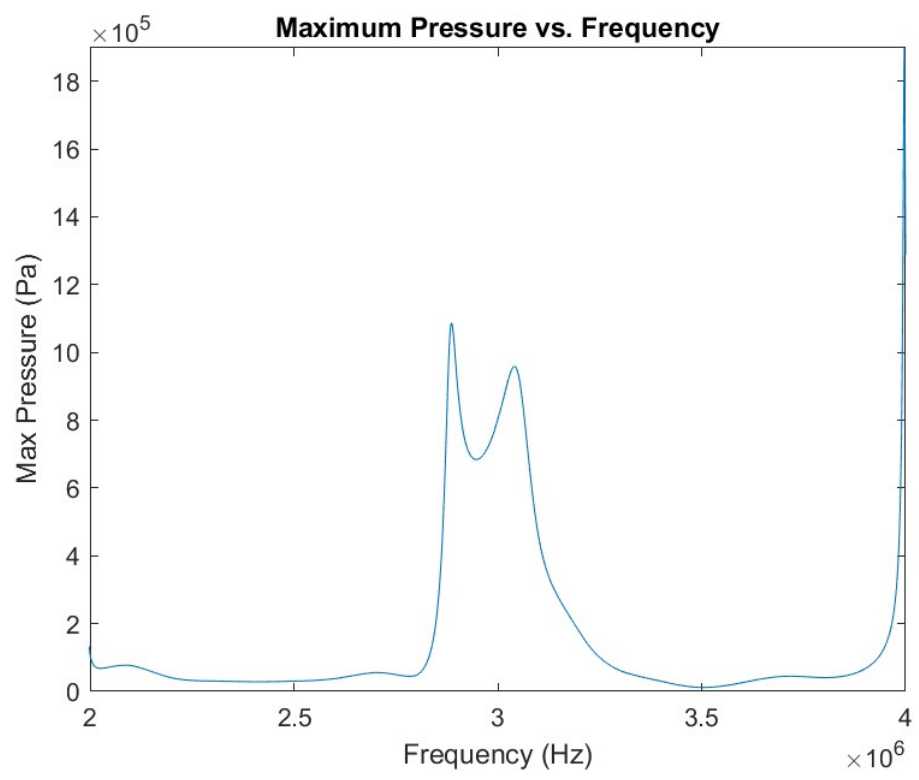

Figure 15: Maximum pressure across the cell across a range of frequencies using interpolation to match voltages with frequencies.

The value corresponding to the peak around $2.9 \mathrm{MHz}$ was used to normalize the curve. This is shown in Figure 16 along with a comparison to Figure 8. 

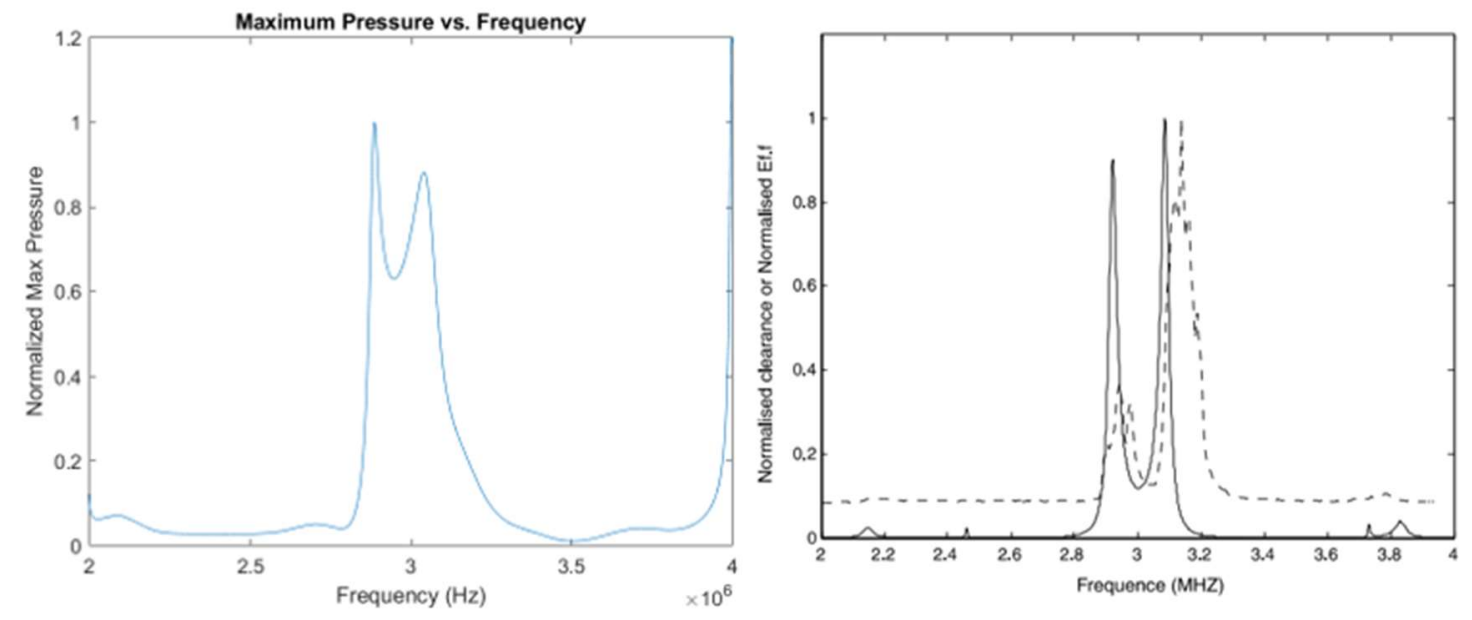

Figure 16: (Left) Normalized max pressure across cell for a range of frequencies. (Right) Figure 8 from Hill study showing normalized clearance

The graph on the left displays the results from the replicated model and is compared to the graph on the right from the Hill study. There is good match showing peaks at frequencies right above and below $3 \mathrm{MHz}$. The larger of the two peaks is opposite in these graphs and the dip between the two peaks isn't as low or detailed, but this is still a good match considering that the interpolation was only over 2100 points and there were no specific transformation ratios used for this graph replication. Assigning a transformation ratio to each frequency step would likely produce a more well-matched result, but this would require a much more complex method than that used in Table 5 .

Considering Figures 14 and 16, the replicated model is a good match to the model developed by Hill et. al. This is reason to translate and develop the model for the microfluidic sonoporation system. 


\section{Microfluidic Sonoporation System Translation}

The first variable that was studied was variation in silicone density. Acoustic pressure profiles for minimum, average, and maximum silicone density values for water and whole blood are shown below in Figures 17-22.

\section{WATER}

\section{BLOOD}
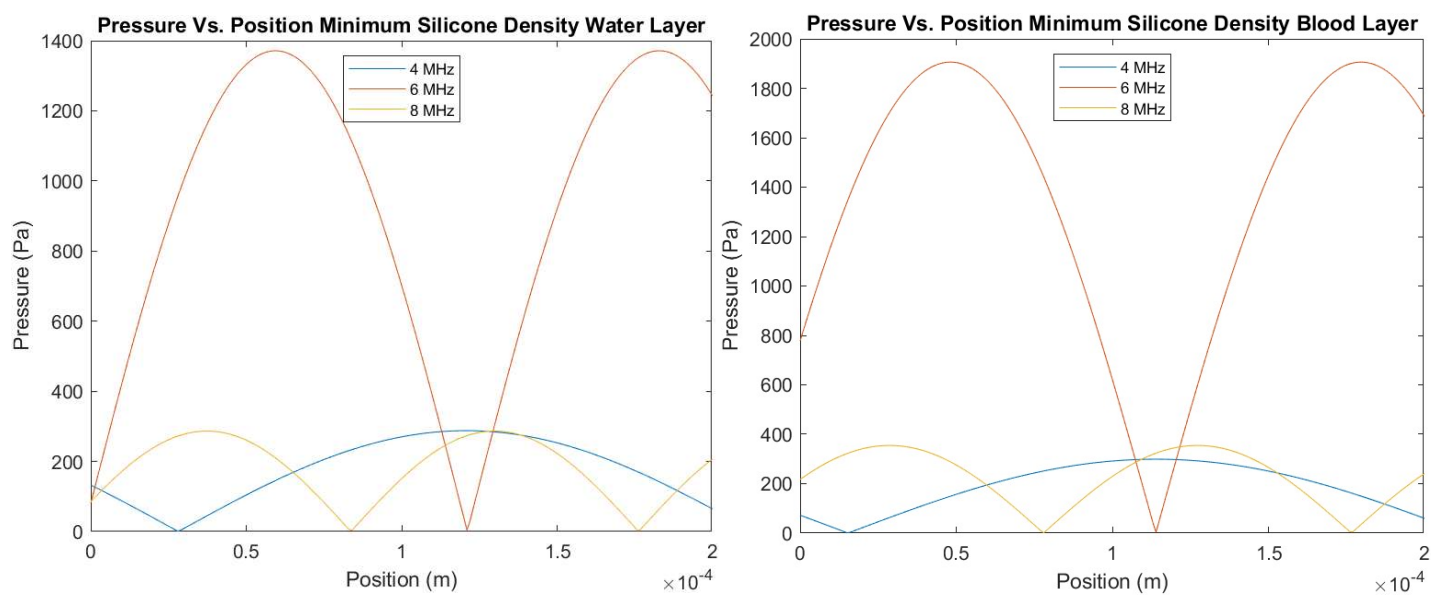

Figure 17: (Left) Minimum silicone density $700 \mathrm{~kg} / \mathrm{m} 3$ water fluid layer

Figure 18: (Right) Minimum silicone density $700 \mathrm{~kg} / \mathrm{m} 3$ blood fluid layer
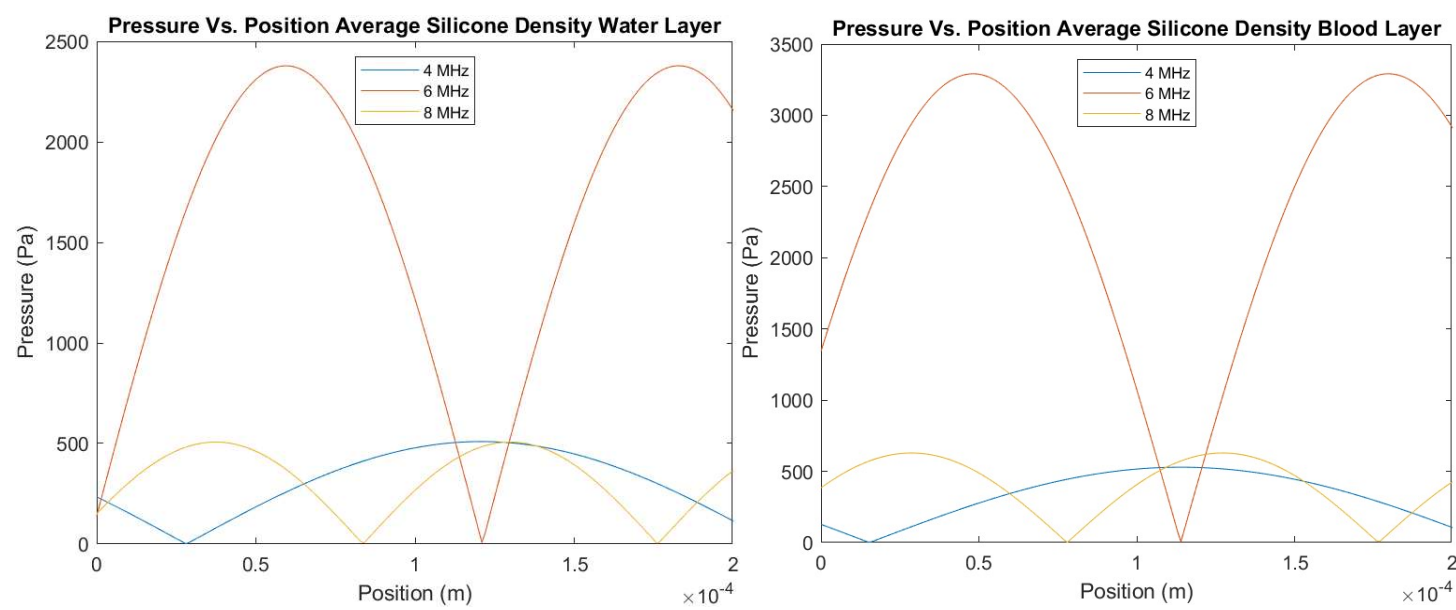

Figure 19: (Left) Average silicone density $1250 \mathrm{~kg} / \mathrm{m} 3$ water fluid layer

Figure 20: (Right) Average silicone density $1250 \mathrm{~kg} / \mathrm{m} 3$ blood fluid layer 

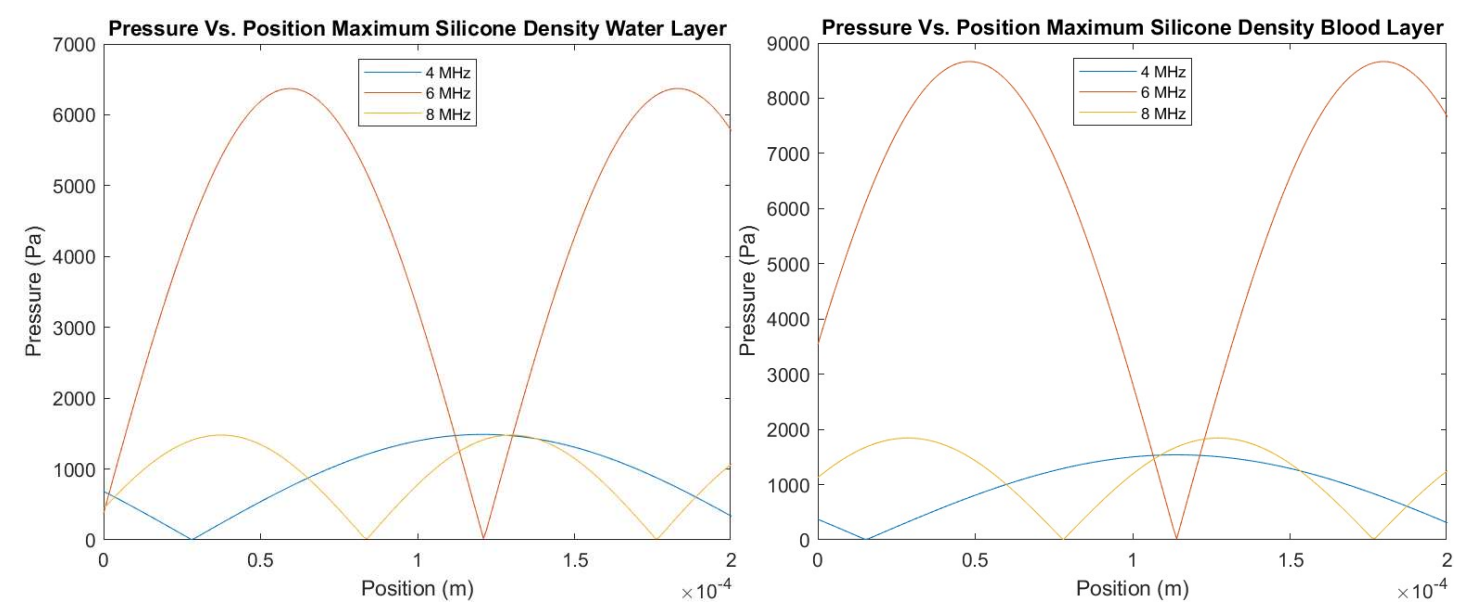

Figure 21: (Left) Maximum silicone density of $3800 \mathrm{~kg} / \mathrm{m} 3$ with water fluid layer

Figure 22: (Right) Maximum silicone density of $3800 \mathrm{~kg} / \mathrm{m} 3$ with blood fluid layer

The number of high-pressure and low-pressure nodes within the microchannel for each frequency, independently, are the same between charts. The locations of these nodes are relatively the same across all densities as well, except for a slight difference in location between the water and blood charts. The range of pressure magnitudes across the charts vary, but all lie within the range of a few thousand pascals. For each chart, the 6 MHz frequency has a much larger magnitude than the 4 and $8 \mathrm{MHz}$ frequencies, which remain very close to each other in maximum pressure values. Between water and blood, the maximum pressure magnitude of the $6 \mathrm{MHz}$ frequency increased greatly in comparison to the other frequencies when using blood parameters. Between the 4 and 8 MHz frequencies, using blood parameters caused the $8 \mathrm{MHz}$ frequency's max-pressure nodes to shift above those of the $4 \mathrm{MHz}$. Increasing silicone density shows an increase in pressure across the cell, the same as increasing fluid density appears to increase pressure across the cell. 
The second variable studied was the variation in silicone acoustic velocity.

Acoustic pressure profiles for minimum, average, and maximum silicone acoustic velocity values for water and whole blood are shown below in Figures 23-28. The title of each chart has SoS (speed of sound) in the title as a replacement for acoustic velocity.

WATER

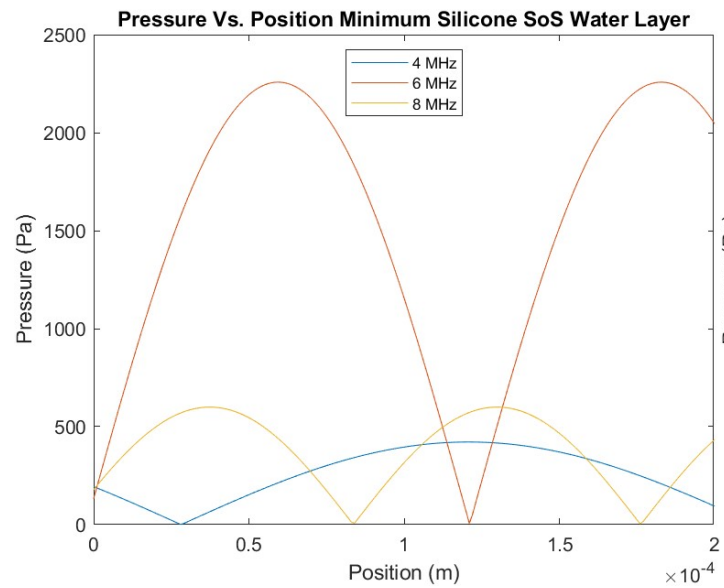

\section{BLOOD}

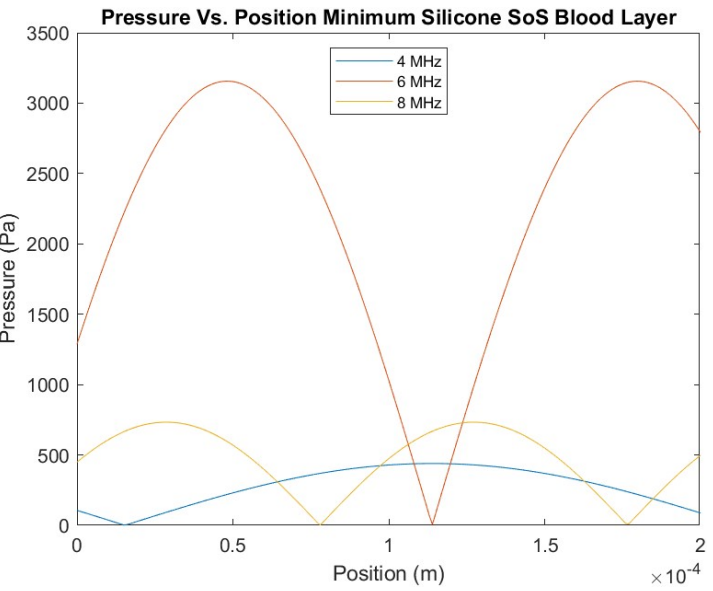

Figure 23: (Left) Minimum silicone speed of sound of $960 \mathrm{~m} / \mathrm{s}$ with water fluid layer

Figure 24: (Right) Minimum silicone speed of sound of $960 \mathrm{~m} / \mathrm{s}$ with blood fluid layer
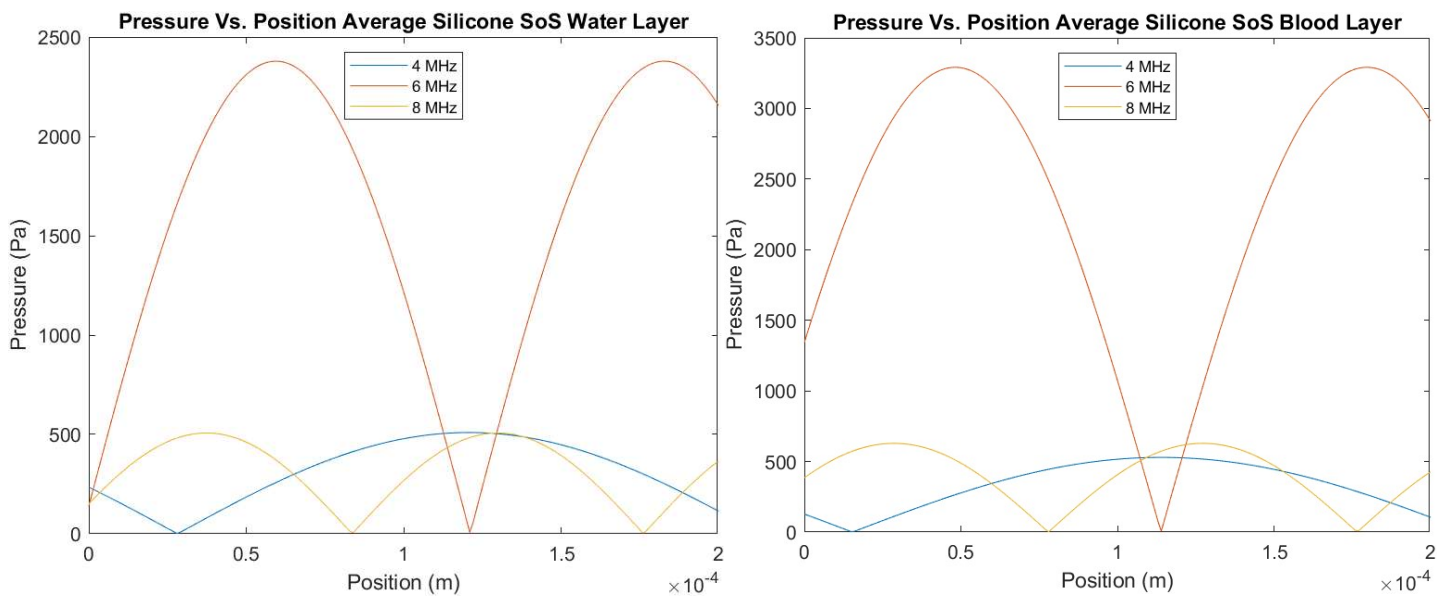

Figure 25: (Left) Average silicone speed of sound of $1035 \mathrm{~m} / \mathrm{s}$ with water fluid layer

Figure 26: (Right) Average silicone speed of sound of $1035 \mathrm{~m} / \mathrm{s}$ with blood fluid layer 

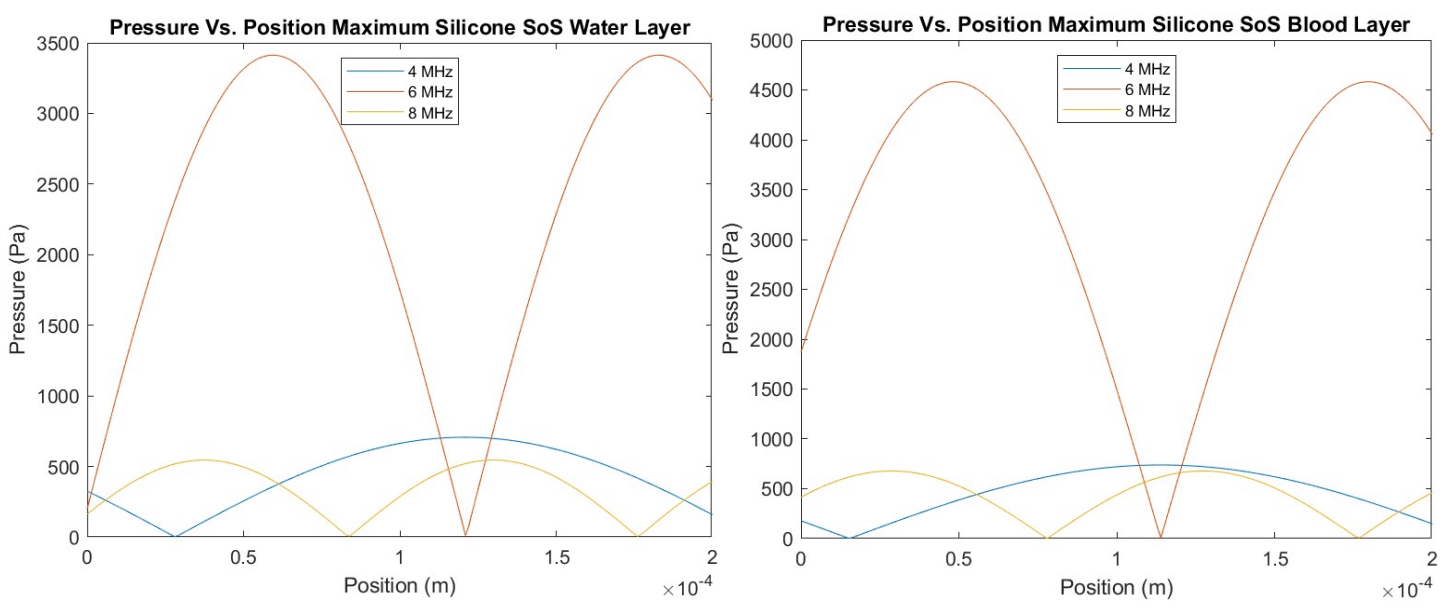

Figure 27: (Left) Maximum silicone speed of sound of $1110 \mathrm{~m} / \mathrm{s}$ with water fluid layer Figure 28: (Right) Maximum silicone speed of sound of $1110 \mathrm{~m} / \mathrm{s}$ with blood fluid layer

Similarly, to Figures 17-22, the number of max-pressure and low-pressure nodes predicted by the model within the cell is the same between charts. The $6 \mathrm{MHz}$ frequency still shows greater pressure magnitudes across the cell, indicating strong resonant behavior at that frequency. The scale on the y-axis is much less varied when changing the acoustic velocity of the silicone layer; three charts have the same maximum pressure on the y-axis and the other three are within 1500 pascals. The variation between 4 and 8 $\mathrm{MHz}$ frequency maximum pressure node values is much less patterned in the charts above. The 4 and $8 \mathrm{MHz}$ frequencies switch between having the larger max-pressure node magnitude between water and blood and across the silicone SoS values randomly. In these charts as in the previous ones, switching between water and blood increased the acoustic pressure for the $6 \mathrm{MHz}$ frequency significantly and the $8 \mathrm{MHz}$ frequency slightly. 
The third variable studied was the variation in PDMS acoustic velocity. Acoustic pressure profiles for minimum, average, and maximum PDMS acoustic velocity values for water and whole blood are shown below in Figures 29-34.

WATER

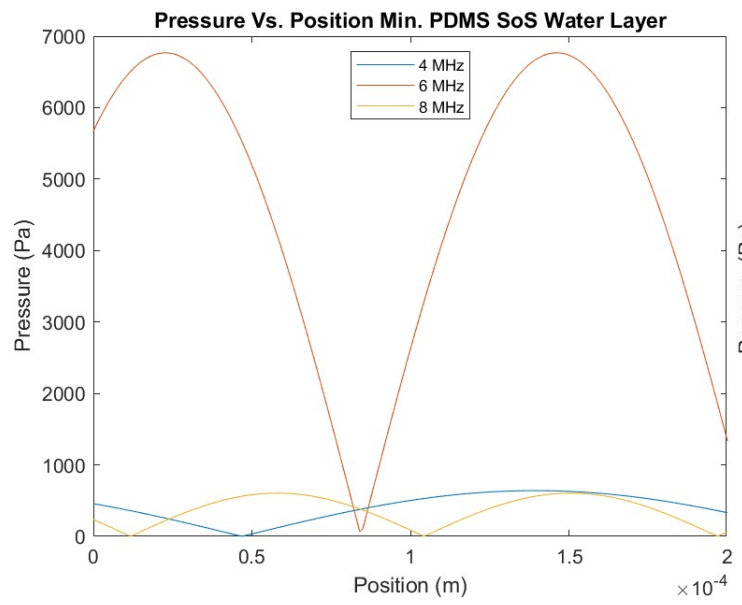

\section{BLOOD}

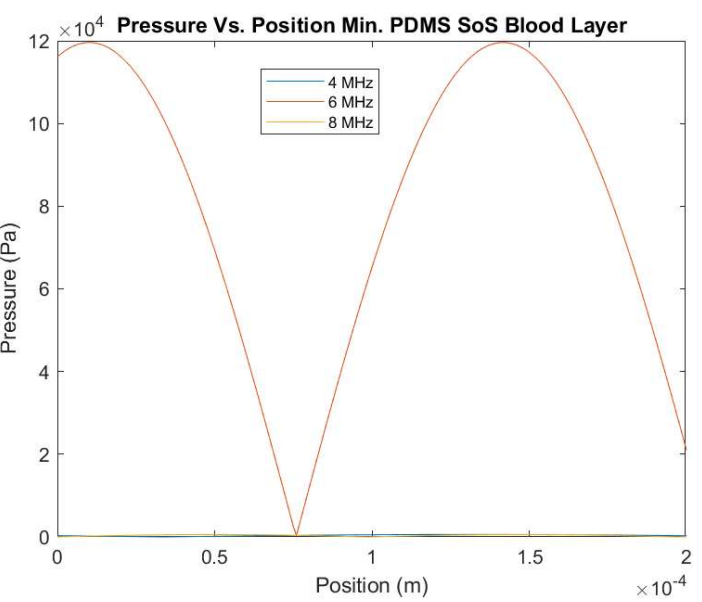

Figure 29: (Left) Minimum PDMS speed of sound of $1076.5 \mathrm{~m} / \mathrm{s}$ with water fluid layer

Figure 30: (Right) Minimum PDMS speed of sound of $1076.5 \mathrm{~m} / \mathrm{s}$ with blood fluid layer
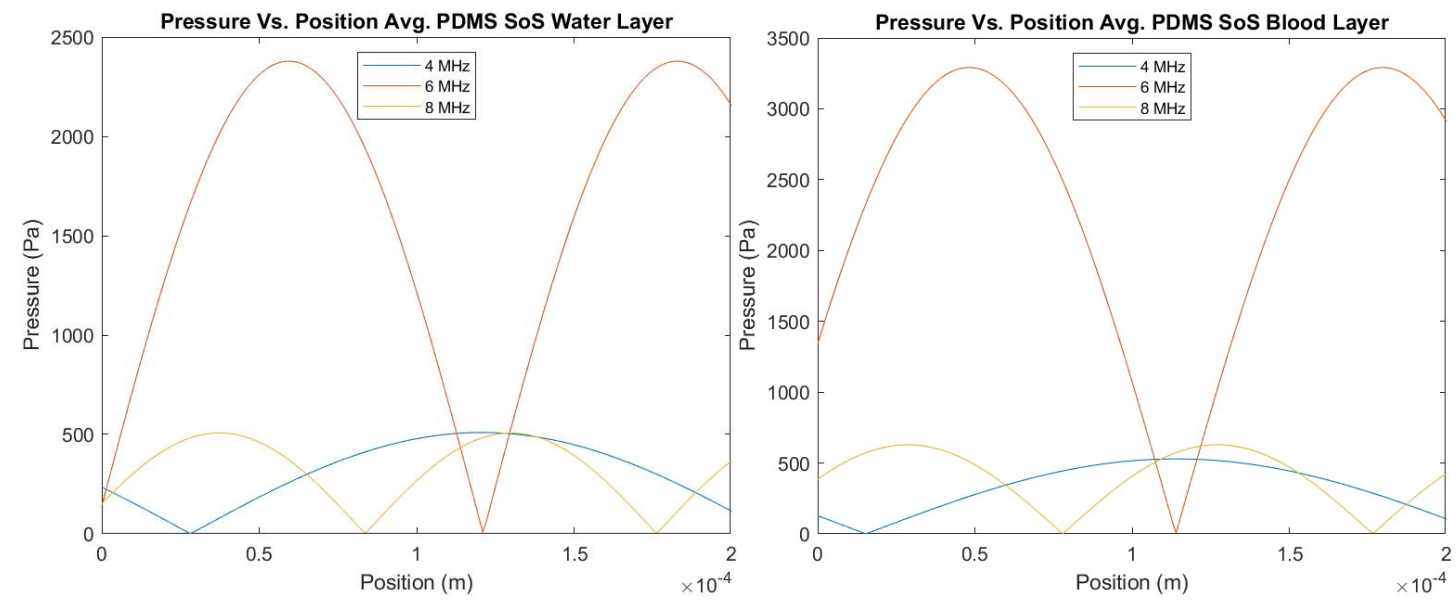

Figure 31: (Left) Average PDMS speed of sound of $1119 \mathrm{~m} / \mathrm{s}$ with water fluid layer

Figure 32: (Right) Average PDMS speed of sound of $1119 \mathrm{~m} / \mathrm{s}$ with blood fluid layer 

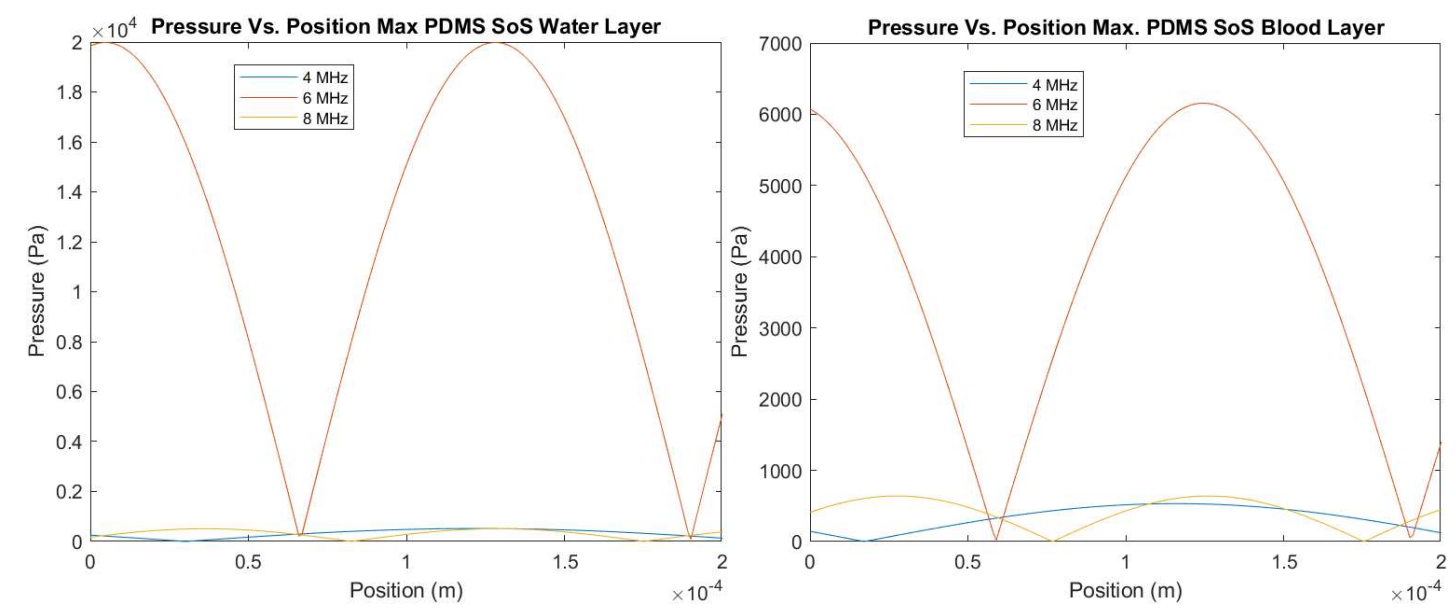

Figure 33: (Left) Maximum PDMS speed of sound of $1250 \mathrm{~m} / \mathrm{s}$ with water fluid layer Figure 34: (Right) Maximum PDMS speed of sound of $1250 \mathrm{~m} / \mathrm{s}$ with blood fluid layer

The charts above show much larger variation than the charts developed for the silicone investigation. The most prominent feature is once again the $6 \mathrm{MHz}$ frequency showing resonant behavior and having the largest pressure magnitudes across the cell as compared to the other two frequencies. The number of pressure nodes stays the same for each frequency in the minimum and average SoS value charts, but when the model is evaluated at the maximum PDMS acoustic velocity the $6 \mathrm{MHz}$ frequency develops an additional low-pressure node at the far end of the cell and the high-pressure node nearer to the transducer gets shifted out of the microchannel. Between water and blood, the pressure magnitude increased when using blood parameters for the $6 \mathrm{MHz}$ frequency except in the maximum PDMS SoS charts. When PDMS is at its maximum acoustic velocity, the pressure within the cell for the $6 \mathrm{MHz}$ frequency is higher for a water like fluid layer as opposed to blood. The large changes that occur when PDMS is at maximum value indicates one of two things: this reported value is incorrect or the acoustic velocity of the reflector layer has a large impact on the pressure profile within the cell. The 4 and 
$8 \mathrm{MHz}$ frequencies behaved similar to each other throughout adjustments and did not vary much in pressure magnitude or nodal positioning, except for one instance where the $8 \mathrm{MHz}$ nodal position was shifted to the right for the minimum SoS value with a water layer.

The fourth variable investigated was layer thickness. Each iteration of the model is evaluated at average values for each material parameter using a water layer, while the thicknesses are doubled or halved for one layer at a time. Figures 35-37 show the variation in silicone layer thickness, Figures 38-40 show the variation in glass layer thickness, and Figures 41-43 show the variation in PDMS layer thickness.

\section{Silicone Thickness Variation:}
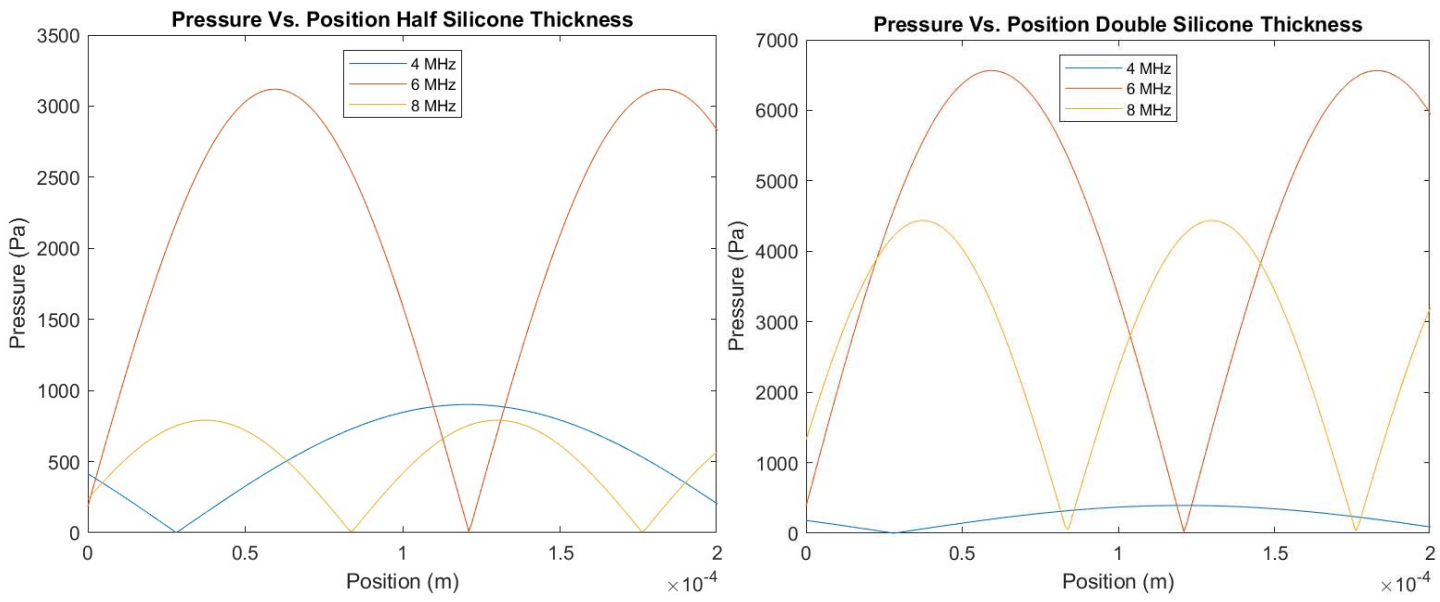

Figure 35: (Left) Half silicone layer thickness of $0.5 \mathrm{~mm}$ with water fluid layer

Figure 36: (Right) Double silicone layer thickness of $2 \mathrm{~mm}$ with water fluid layer 


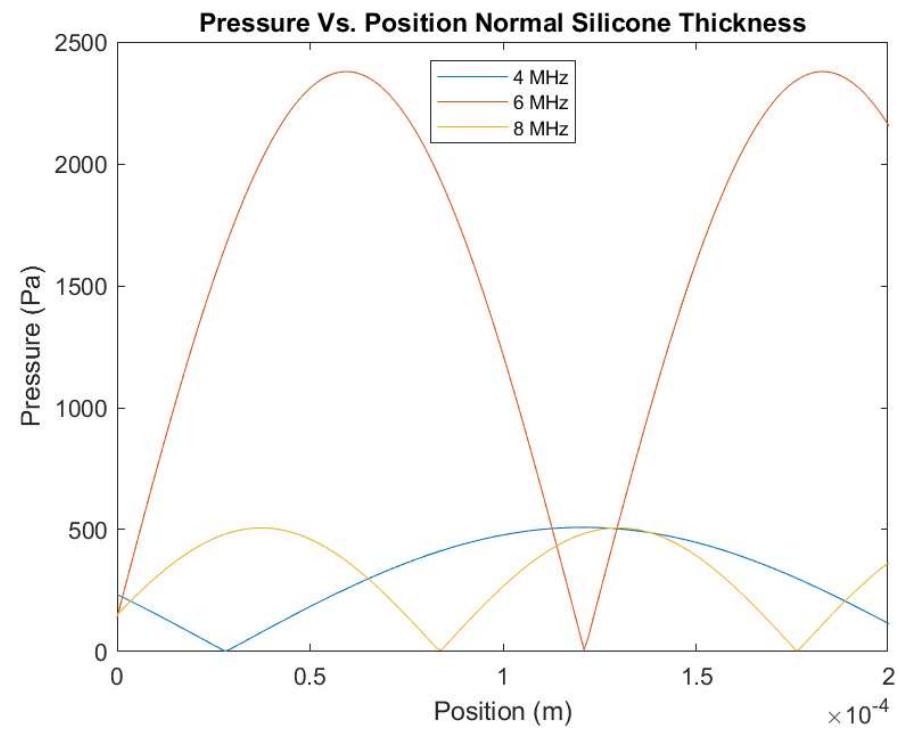

Figure 37: Normal silicone layer thickness of $1 \mathrm{~mm}$ with water fluid layer

\section{Glass Thickness Variation:}
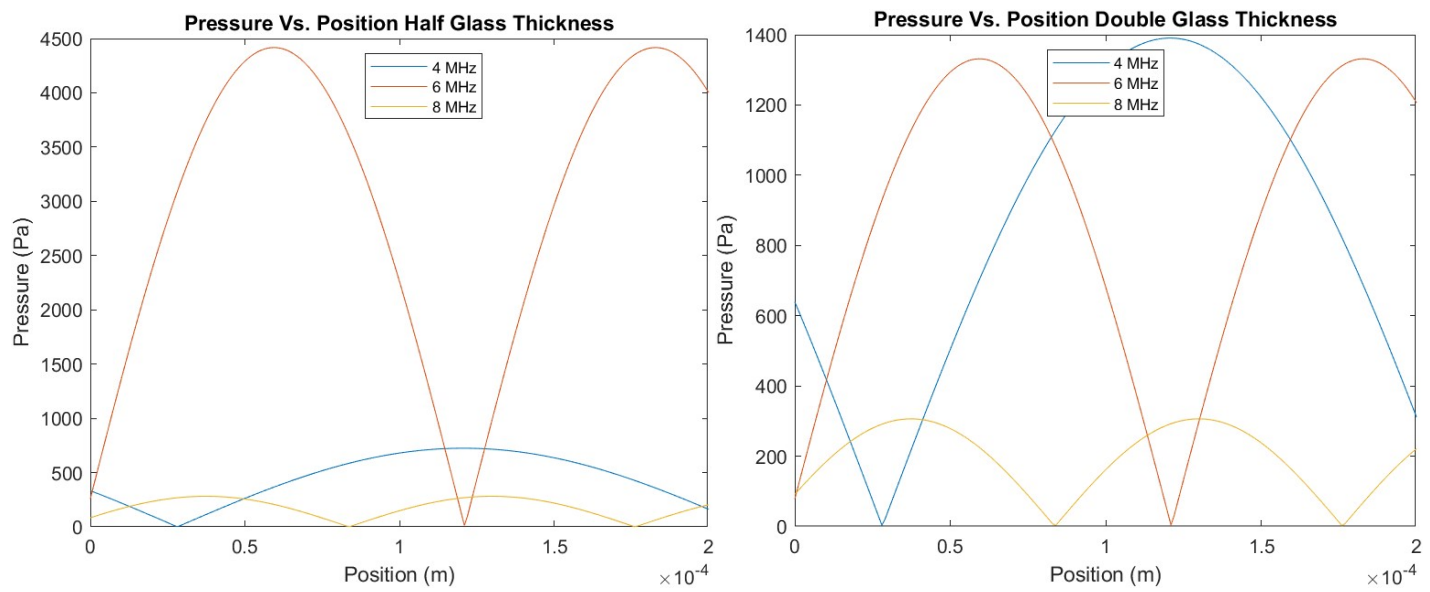

Figure 38: (Left) Half glass thickness of $0.5 \mathrm{~mm}$ with water fluid layer

Figure 39: (Right) Double glass layer thickness of $2 \mathrm{~mm}$ with water layer 


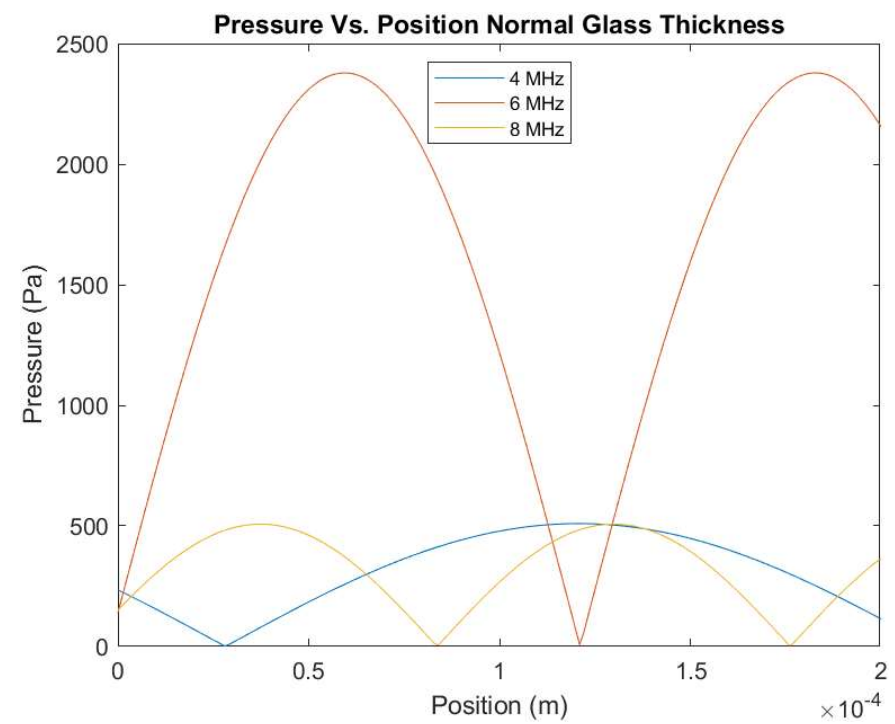

Figure 40: Normal glass layer thickness of $1 \mathrm{~mm}$ with water fluid layer

\section{PDMS Thickness Variation:}
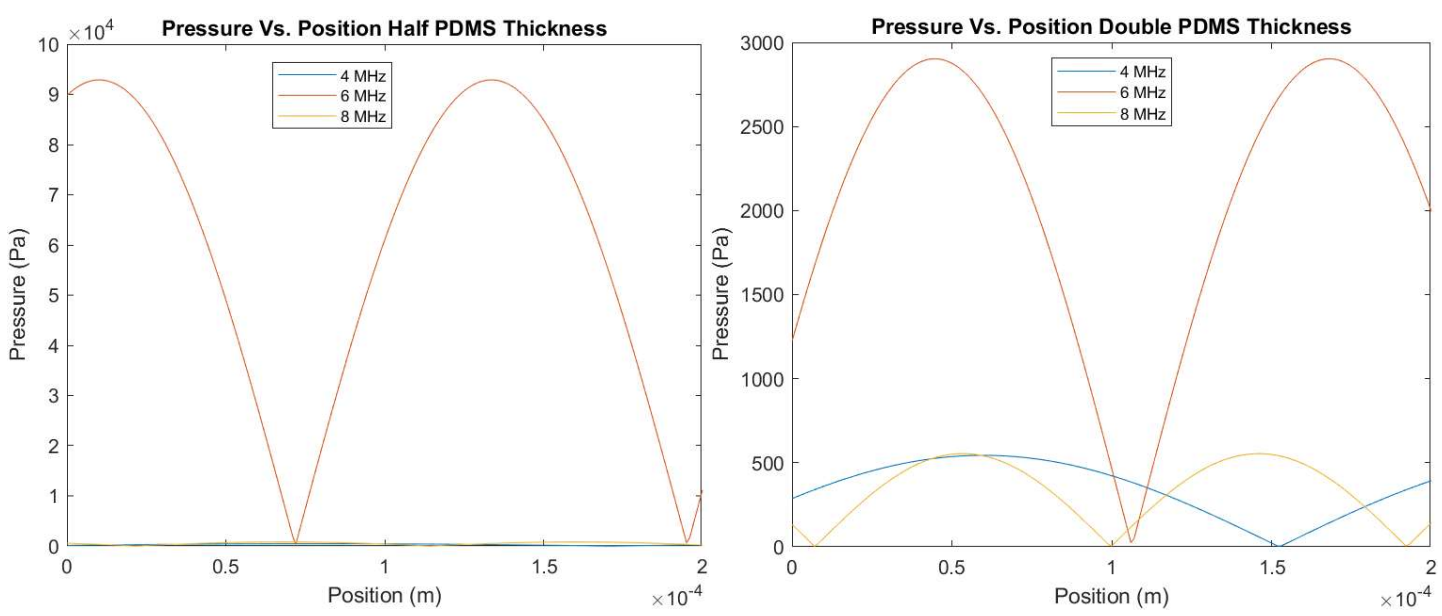

Figure 41: (Left) Half PDMS layer thickness of $2 \mathrm{~mm}$ with water fluid layer

Figure 42: (Right) Double PDMS layer thickness of $8 \mathrm{~mm}$ with water fluid layer 


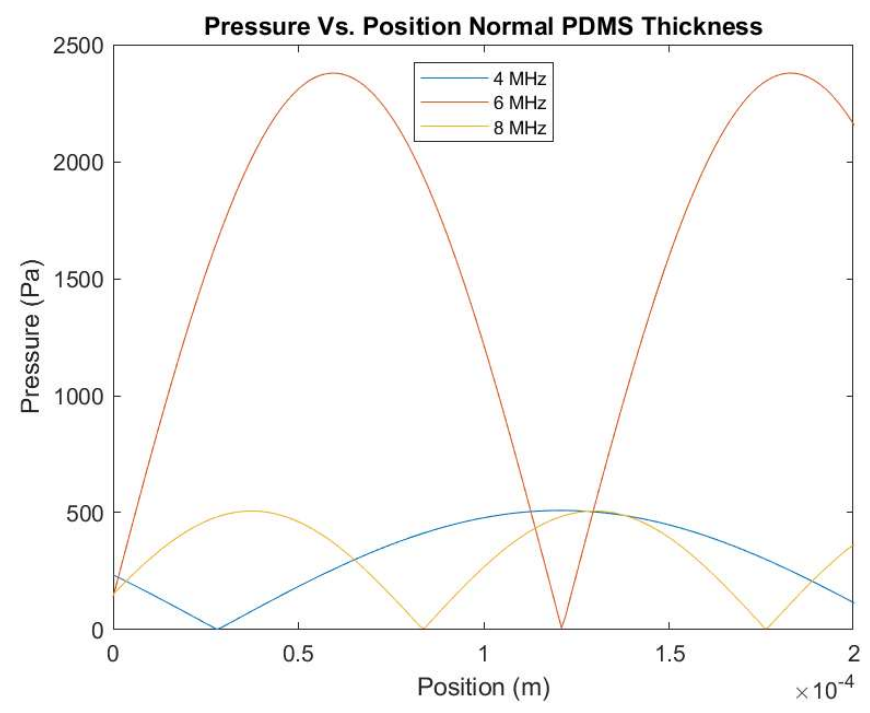

Figure 43: Normal PDMS thickness of $4 \mathrm{~mm}$ with water fluid layer

When varying thickness for the silicone (matching) and glass layers, the nodal positioning remains the same for all frequencies. However, when varying the thickness for the PDMS (reflector layer) there was a large shift in nodal positioning for all frequencies. For the silicone layer, both halving and doubling the thickness increased the maximum pressure for both 6 and $8 \mathrm{MHz}$. There was a significant jump in pressure for the $8 \mathrm{MHz}$ frequency when doubling the matching layer thickness. For the glass layer, doubling the thickness decreased the maximum pressure for every frequency except 4 $\mathrm{MHz}$, which greatly increased. Halving the glass thickness nearly doubled the $6 \mathrm{MHz}$ pressure while having little effect on the other frequencies. Halving the PDMS thickness greatly increased the pressure of the $6 \mathrm{MHz}$ profile while nearly eliminating the pressure of the other two frequency profiles. This change also shifted the low-pressure node of the $6 \mathrm{MHz}$ profile from right of cell center to the left of it, which has only been seen in iterations of the model where the acoustic velocity of the PDMS layer was altered. Doubling PDMS thickness had little effect other than to slightly increase pressure for the 
$6 \mathrm{MHz}$ profile and shift the nodal positioning of the $8 \mathrm{MHz}$ profile more symmetrically across the cell.

The replication of Figure 8 for the microfluidic system required finding the maximum pressure across the cell for the range of calibrated values from the transducerhydrophone calibration. Figures 44 and 45 show the results using interpolation of voltages for each frequency step with both water and blood fluid layer parameters.
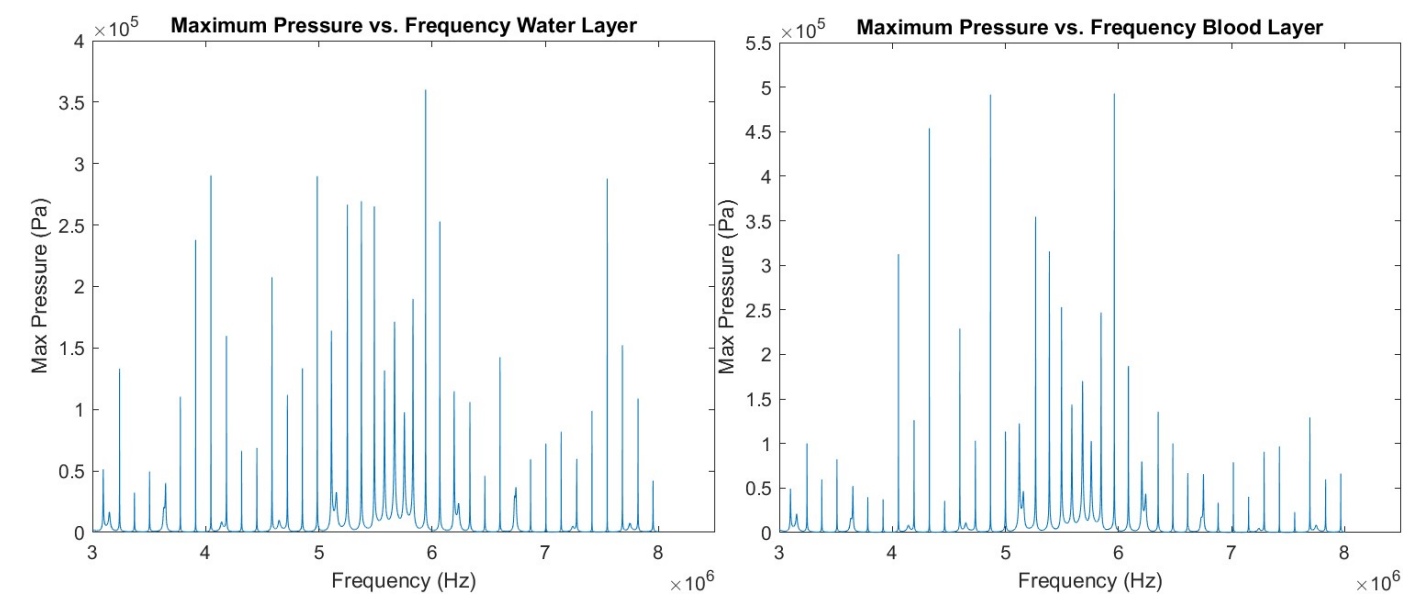

Figure 44: (Left) Maximum pressure vs. frequency for interpolated range of frequencies from 3-8 $\mathrm{MHz}$ water layer

Figure 45: (Right) Maximum pressure vs. frequency for interpolated range of frequencies from 3-8 $\mathrm{MHz}$ blood layer

Normalized charts for the figures above were created by dividing by the largest pressure in each graph and are shown in Figures 46 and 47. 

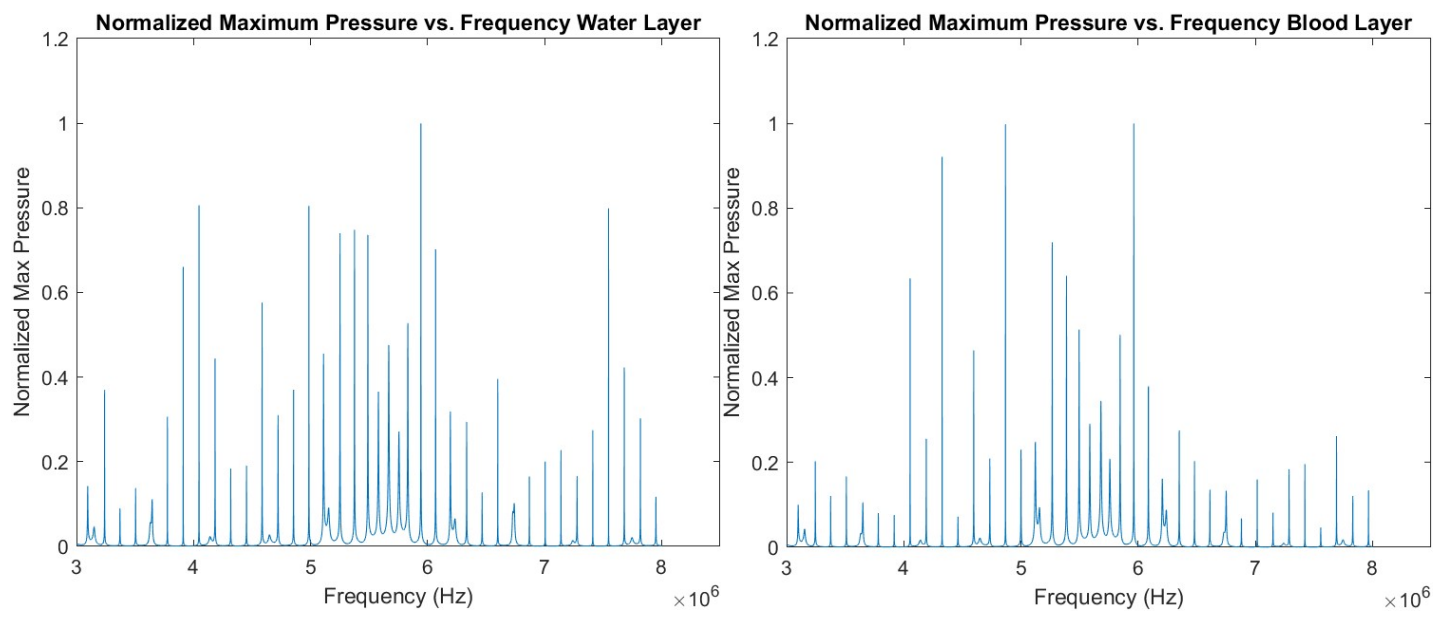

Figure 46: (Left) Normalized max pressure vs. frequency water layer

Figure 47: (Right) Normalized max pressure vs. frequency blood layer

These graphs show resonant spikes at multiple frequencies throughout the cell. The most prominent resonances occur around 4, 5, 6, and 7.5 $\mathrm{MHz}$ for the water layer while they occur between $4-5 \mathrm{MHz}$ and at $6 \mathrm{MHz}$ for the blood layer. For these results to agree with Figures 17-43, the largest resonance is expected to occur around $6 \mathrm{MHz}$ with smaller resonances around 4 and $8 \mathrm{MHz}$. These results agree with the figures and also correspond with the hydrophone calibration which shows the transducer having the largest resonance at $6 \mathrm{MHz}$.

\section{Experimental Trials}

The experimental trials measured the cellular uptake of calcein (a fluorescent agent) into RBCs at different ultrasound frequencies. The results of this experiment are displayed below in Figure 48 . 


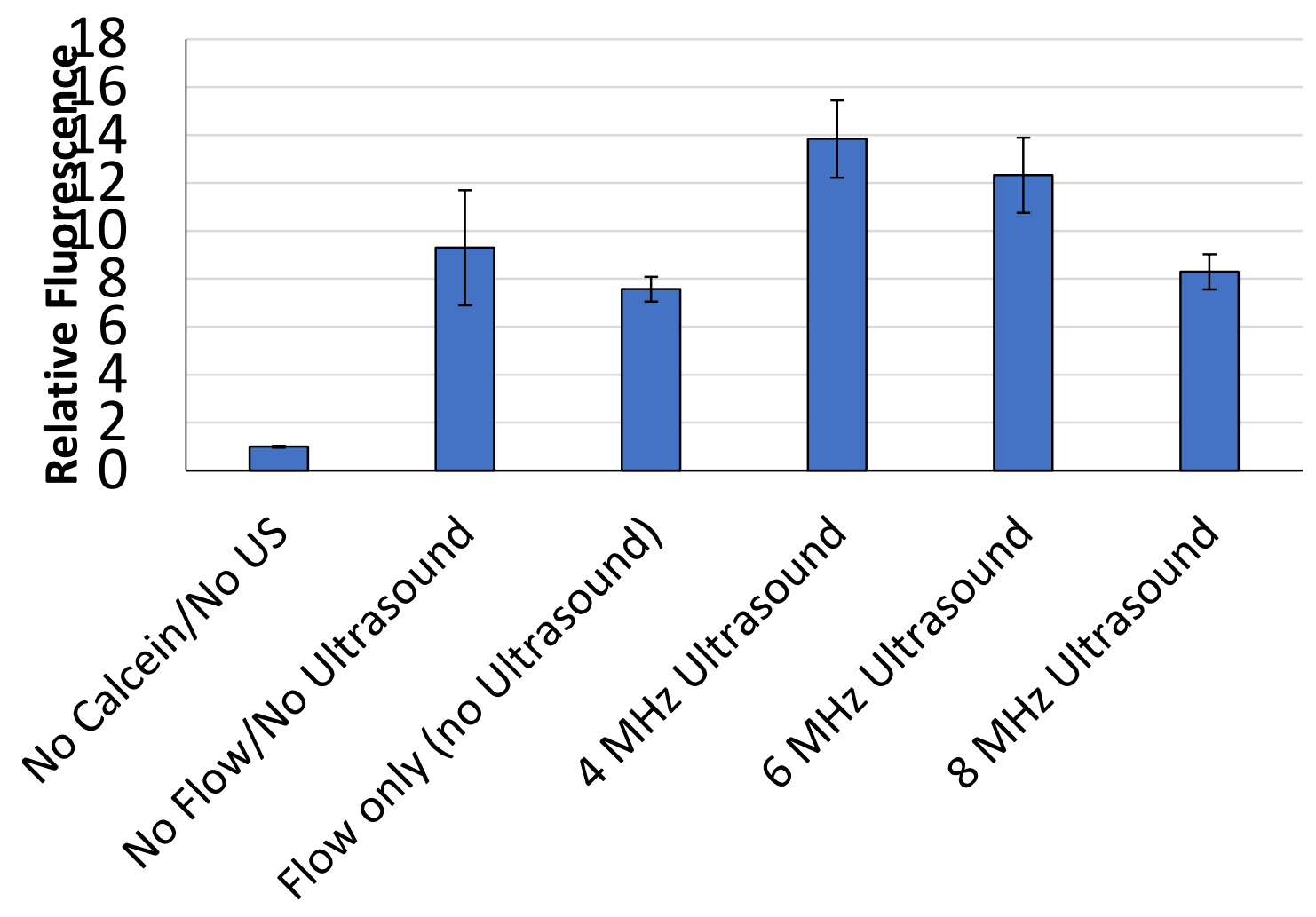

Figure 48: Relative fluorescence within RBCs after being treated with different system conditions and at different driving frequencies

The relative fluorescence is greatest at the $4 \mathrm{MHz}$ frequency and followed closely by the $6 \mathrm{MHz}$ frequency. The $8 \mathrm{MHz}$ frequency shows even lower cellular uptake than the control group with no flow or ultrasound. A 2-way ANOVA test was conducted $(p=$ 0.04) that determined that the $4 \mathrm{MHz}$ ultrasound group was statistically different from the $8 \mathrm{MHz}$ ultrasound group. An additional ANOVA was conducted $(\mathrm{p}=0.003)$ that found the $4 \mathrm{MHz}$ group was statistically different from the flow only (no ultrasound) group as well. These results do not correlate with the modelled data from Figures 17-43 in that the $6 \mathrm{MHz}$ driving frequency shows the largest pressures across the cell while the $4 \mathrm{MHz}$ frequency shows the highest cellular uptake during experimental trials. 


\section{CONCLUSIONS}

The Hill study replication proved to be a valid model to translate to the microfluidic sonoporation system based on the replicated graphs. Improving the replicated model would require more information about the electrical characteristics of the transducer used in the Hill study so that specific, rather than estimated, transformation ratios could be determined.

The translation of the Hill model to the microfluidic sonoporation system showed results that did not agree with experimental trials. Most significantly, the model predicted the $6 \mathrm{MHz}$ profile to have the greatest pressure magnitude across the cell for multiple variations in parameters. This does not correlate with the $4 \mathrm{MHz}$ frequency having the greatest uptake in fluorescence during experimental trials. Large resonant behavior does not appear to be directly related to transfection effectiveness in this sonoporation system, although further experimental trials are necessary to confirm this hypothesis.

The model predicted node spacing correctly. The spacing of nodes is expected to a multiple of the half wavelength of the driving frequency, so at higher frequencies the spacing between nodes should be smaller. This can be seen in the model as the $8 \mathrm{MHz}$ frequency has the smallest node spacing, followed by 6 and then $4 \mathrm{MHz}$. 
The actual location of the nodes rarely varied with parameter alteration, except when the PDMS (reflector layer) is altered. As this is the case, the impact of the reflector layer on the acoustic profile seems to be significant. A possible explanation for the variation in node spacing and positioning could be the effect of interference patterns caused by waves reflected off the PDMS layer. When acoustic waves hit the reflector, a phase shift occurs and the reflected waves interfere destructively with the transmitted waves. This interference can eliminate or space out low- and high-pressure regions and create a more even pressure profile across the cell as seen with the $4 \mathrm{MHz}$ frequency profile in many of the figures. Depending on the phase shift, this interference could also cause node shifts as seen in the 6 and $8 \mathrm{MHz}$ frequencies when altering PDMS parameters.

The investigation into parameter alteration showed some interesting results. Except for one iteration of the model, the pressure increased for the $6 \mathrm{MHz}$ profile when using blood parameters for the fluid layer. A denser fluid within the microchannel predicts an increase in the maximum pressure of the standing waves for the resonant frequency. The variation in silicone acoustic velocity showed little impact on the magnitude of pressures within the cell or the position and number of nodes. Increasing silicone density appears to increase the pressure across the cell, just as increasing fluid layer density increases pressure across the cell. Varying the layer thickness had a significant impact across all tested layers. Doubling and halving the thickness of different layers changed the magnitudes of the pressure profiles for all frequencies, but the most significant figure is the doubling of the glass layer thickness. When doubling the glass layer thickness from 1 to $2 \mathrm{~mm}$, the pressure profile across the cell correlates strongly 
with experimental results. The $4 \mathrm{MHz}$ frequency has the largest pressure followed by the $6 \mathrm{MHz}$ frequency, and the $8 \mathrm{MHz}$ frequency shows very low pressure. Changing the thickness of the glass layer is an important parameter for investigation in future works. Additionally, the effects of changing matching layer thickness are significant. Shifts in pressure for the underperforming frequencies are apparent when the matching layer thickness is altered.

The maximum pressure figures (Figures 44-47) seem to correlate well with results predicted by the model but not with results seen in experimental trials. This is the result of how the model was developed. The modeled data correlates well with other modeled data because it is based on the transducer calibration. The transducer shows resonance at $6 \mathrm{MHz}$ during calibration and this effect translates to high pressures within the model. Figures 44-47 are choppy, but a greater sampling rate during transducer calibration may help to improve the interpolation process for the unknown frequency voltages. However, it is possible there are resonances at all of these predicted frequencies and interpolation will only further refine the exact frequency these occur at.

Overall, this model could be used in the future for predicting the acoustic pressure profile across the microfluidic channel. For this model to be used in the future, more iterations of the model need to be evaluated with greater precision of parameter variation. The results of these iterations need to be compared to experimental trials to determine the exact cell parameters which predict real-world behavior. Once the model is improved and the actual cell parameters are determined, the model could be used to design the desired ultrasound pressure profile across the cell for specific applications. 


\section{LIMITATIONS}

The Hill paper had a few limitations that were taken into account. The model assumes that the acoustical characteristics within the resonator are dominant in the thickness direction of the transducer. This means that the model neglects to consider shearing affects and models the acoustic waves as longitudinal waves. Additionally, there was a lack of information about the electrical characteristics of the transducer, which required estimation of numerous values used in the model validation. The method for determining transformation ratios is valid but could be improved if the modeled voltage data was provided by the authors for the frequencies being investigated. The authors included values for "q factors" in their diagrams saying they represented losses within the model, but how these factors were included is unknown and not present in the model validation. Additionally, the authors used a method to estimate cell performance based on ultrasonic particle separation, whereas the microfluidic sonoporation system is mainly used for cell transfection or molecular delivery.

The limitations of the translated model arose from limitations with the Hill model as well as some lack of information about the microfluidic chip parameters. The translated model also only considered effects in the thickness direction, but as the flow profile is fairly laminar this may not have much of an impact on the microfluidic channel. The electrical characteristics of the PZT transducer for the microfluidic device were not 
exactly known either. The impedance of the transducer when shorted, as well as the transformation ratio at each frequency, was unknown. Exact measurements of the layer thicknesses were not made either. A ruler was used to estimate layer thickness whereas a laser-based method may be more appropriate for the scale of the layers. The complexity of the fluid layer was also a limitation of the developed model. Using water and blood parameters to estimate the fluid density is not exact and has a significant impact as seen in the variation of results between water and blood layers. Lastly, the calibration of the transducer was conducted within a free-field of water and limited to a few data points which could have affected the voltages used to generate both the pressure profile and maximum pressure charts. 


\section{FUTURE WORKS}

Considering the results and limitations of this study, some areas for improvement and investigation are suggested. The model should be improved by iterating through higher precision of material parameters within the ranges suggested by the tables. These iterations should be compared to experimental trials to determine which parameters match most closely with those of the microfluidic chip. A more in-depth investigation into thickness variation is suggested to determine if the desired pressure profile can be generated across the cell. The measurement of layer thicknesses needs to be conducted in a more precise manner for more accurate results to be predicted. A method for calibrating the transducer voltage output (and terminal impedance when shorted) while connected to the cell should be created to develop a smoother and more accurate set of data. The complex fluids that flow through the microfluidic device should have their densities determined more accurately as well. 


\section{REFERENCES}

1. Douglas, Kimberly L. "Toward Development of Artificial Viruses for Gene Therapy: A Comparative Evaluation of Viral and Non-Viral Transfection.” Biotechnology Progress, vol. 24, no. 4, 2008, p. 871.

2. Murphy, Emily M. 'NON-VIRAL TRANSFECTION EFFICIENCIES FOR THE ADVANCEMENT OF CAR-T THERAPY.” August 2019. University of Louisville. Masters of Bioengineering Thesis

3. Yang Y, et al. "Mechanisms Underlying Sonoporation: Interaction between Microbubbles and Cells.” Ultrasonics Sonochemistry, vol. 67, 2020, pp. 105096105096., doi:10.1016/j.ultsonch.2020.105096.

4. Lamprecht M, and Dansereau C. "Car T-Cell Therapy: Update on the State of the Science.” Clinical Journal of Oncology Nursing, vol. 23, no. 2, 2019, pp. 6-12., doi:10.1188/19.CJON.S1.6-12.

5. Shah, Nirali N, and Terry J Fry. "Mechanisms of Resistance to Car T Cell Therapy." Nature Reviews Clinical Oncology, vol. 16, no. 6, 2019, pp. 372-385., doi:10.1038/s41571-019-0184-6.

6. Neelapu, Sattva S. "Managing the Toxicities of Car T-Cell Therapy." Hematological Oncology, vol. 37, 2019, pp. 48-52., doi:10.1002/hon.2595. 
7. Hill, Martyn, et al. "Modelling of Layered Resonators for Ultrasonic Separation.” Ultrasonics, vol. 40, no. 1, 2002, pp. 385-392., doi:10.1016/S0041$624 \mathrm{X}(02) 00127-0$.

8. Azhari, Haim. Basics of Biomedical Ultrasound for Engineers. Wiley, 2010.

9. Input impedance equation: L.E. Kinsler, A.E. Frey, A.B. Coppens, J.V. Sanders, Fundamentals of Acoustics, Wiley, New York, 1982.

10. Hawkes, Jeremy J, and W.terence Coakley. "Force Field Particle Filter, Combining Ultrasound Standing Waves and Laminar Flow." Sensors and Actuators B: Chemical, vol. 75, no. 3, 15 May 2001, pp. 213-222., doi:10.1016/s0925-4005(01)00553-6. 


\section{APPENDIX}

\section{Hill Pressure Profile Validation Code:}




Z $S S B=(A I S S B * C S$ Area $) *(($ Z air +

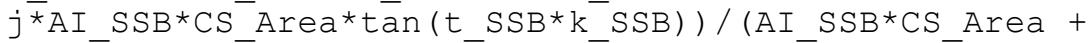

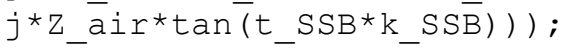

oMaterial Properties for Water

t_water $=250 e-06$;

oThickness of the Water Layer (m)

rho_water $=1000$;

oDensity of Water $(\mathrm{kg} / \mathrm{m} 3)$

c_water $=1500$;

$\mathrm{k}$-water $=$ omega/c water;

ospeed of Sound in Water $(\mathrm{m} / \mathrm{s})$

owavenumber in the Water Layer

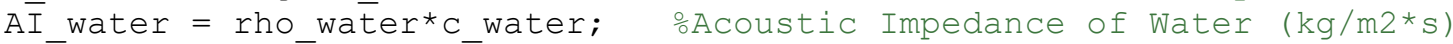

oMechanical Impedance of the Water Layer

Z_water $=($ AI_water*CS_Area $) *((Z$ Z_SB +

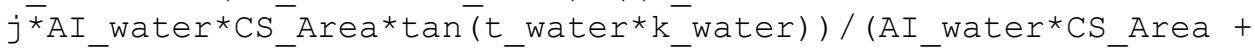

$j * z \_\bar{S} S B * \tan \left(t \_\right.$water*k_water) )) ;

oMaterial Properties for Initial stainless steel Layer

t_SSI = 3.1e-03; $\quad$ oThickness of the Initial SS Layer $(\mathrm{m})$

rho_SSI = 7800; $\quad$ oDensity of Initial SS Layer (kg/m3)

C SSTI = 6200; $\quad$ ospeed of Sound in Initial SS Layer $(\mathrm{m} / \mathrm{s})$

k_SSI = omega/c_SSI; $\quad$ oWavenumber in the Initial SS Layer

$\bar{A} \bar{I}$ SSI $=$ rho_SSI*C_SSI; $\quad$ ofcoustic Impedance of Initial SS Layer

$(\mathrm{kg} / \mathrm{m} 2 * \mathrm{~s})$

oMechanical Impedance of Initial SS Layer

Z_SSI $=($ AI_SSI*CS_Area $) *((Z$ _water +

$j{ }^{\star A} \mathrm{~A}$ _SSI*CS_Area*tan(t_SSI*k_SSI))/(AI_SSI*CS_Area +

$j * z \_\bar{w}$ ater*tan $\left.\left.\left(t \_S S I * k \_\bar{S} S I\right)\right)\right)$;

oMaterial Properties for Epoxy Resin Layer

t_ER = 15e-06; $\quad$ oThickness of the Epoxy Resin Layer (m)

rho_ER = 2000; $\quad$ oDensity of Epoxy Resin Layer $(\mathrm{kg} / \mathrm{m} 3)$

C_E $E \bar{R}=2440 ; \quad$ ospeed of Sound in Epoxy Resin Layer (m/s)

$\mathrm{k}$ ER = omega/C_ER; $\quad$ oWavenumber in the Epoxy Resin Layer

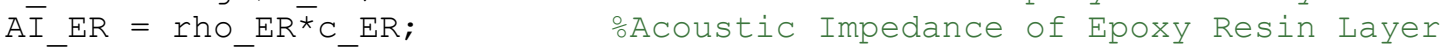

$(\mathrm{kg} / \mathrm{m} 2 * \mathrm{~s})$

oMechanical Impedance of Epoxy Resin Layer

Z_ER $=\left(A I \_E R * C S \_A r e a\right) *((Z$ ZSSI +

$j{ }^{*} A I \_E R * C S$ Area*tan $\left(t \_E R * k\right.$ ER $\left.)\right) /\left(A I E E * C S \_A r e a+\right.$

$\left.j * z_{-} \bar{S} S I * \tan \left(t \_E R * k_{-} E R \overline{)}\right)\right)$;

oMaterial Properties for Silver Electrode Layer

t_SE $=2 e-06 ; \quad$ oThickness of the Silver Electrode Layer

$(\bar{m})$

rho_SE = 10400; $\quad$ oDensity of Silver Electrode Layer (kg/m3)

c $S \bar{E}=3650 ; \quad$ ospeed of Sound in Silver Electrode Layer

$(\bar{m} / \mathrm{s})$

k_SE = omega/C_SE; $\quad$ oWavenumber in the Silver Electrode Layer

$\mathrm{A} \overline{\mathrm{I}}$ SE $=$ rho_SE${ }^{*}{ }_{C}$ SE; $\quad$ ofcoustic Impedance of Silver Electrode

Laȳer $(\mathrm{kg} / \mathrm{m} \overline{2} * \mathrm{~s})$

oMechanical Impedance of Silver Electrode Layer

Z_SE $=\left(A I \_S E * C S \_A r e a\right) *\left(\left(Z \_E R+\right.\right.$

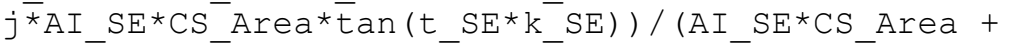

$\left.j * z_{-} \bar{E} R * \tan \left(t_{-} S E * k_{-} S E\right) \overline{)}\right)$;

oForce Generated by Transducer Acting on Silver Electrode Layer

$\mathrm{V}=\operatorname{Voltage}(\mathrm{i}) ; \quad$ oin volts $(\mathrm{V})$

F_transducer $=\left(T R * V * Z \_S E\right) /\left(Z\right.$ transducer $+Z_{-}$SE $)$; 


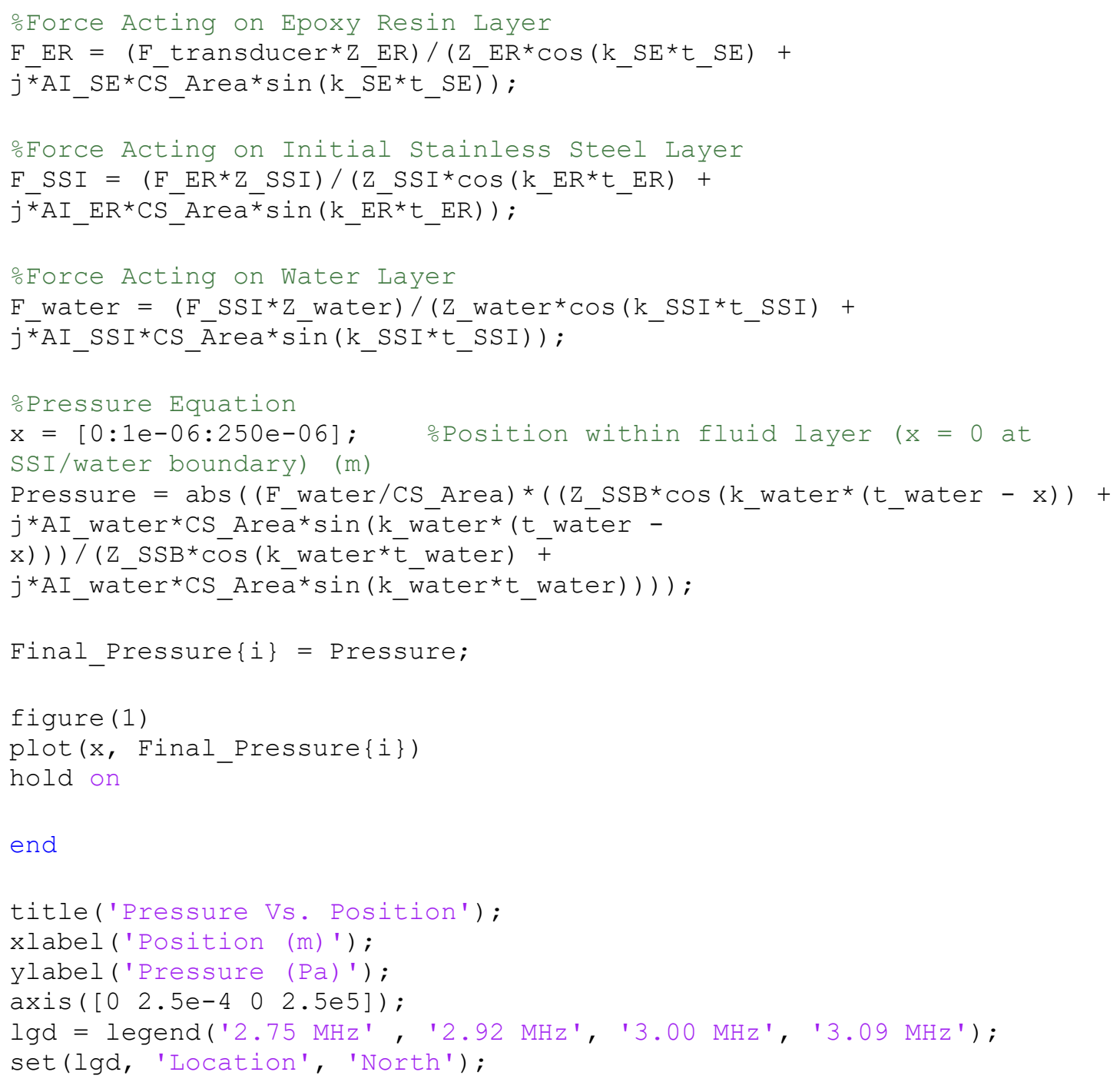

\section{Hill Normalized Clearance Validation Code:}

oAuthor: Chris Holton

oAdvised by: Dr. Jonathan Kopechek

oDr. Kopechek Laboratory University of Louisville

clear all

close all

Voltage $=[1.3,0.25,1.75,1.5,1.4,1.3,1.2,1.1,0.95,0.3,1.075$, 1.95, 1.3, 1.15, 1.0, 0.85, 0.45, 1.15, 1.9, 1.4, 1.3]; oVoltage across Transducer terminals

phi $=[1]$

oTransformation Ratio for

Transducer, frequency dependent. Different constant for each driving frequency 


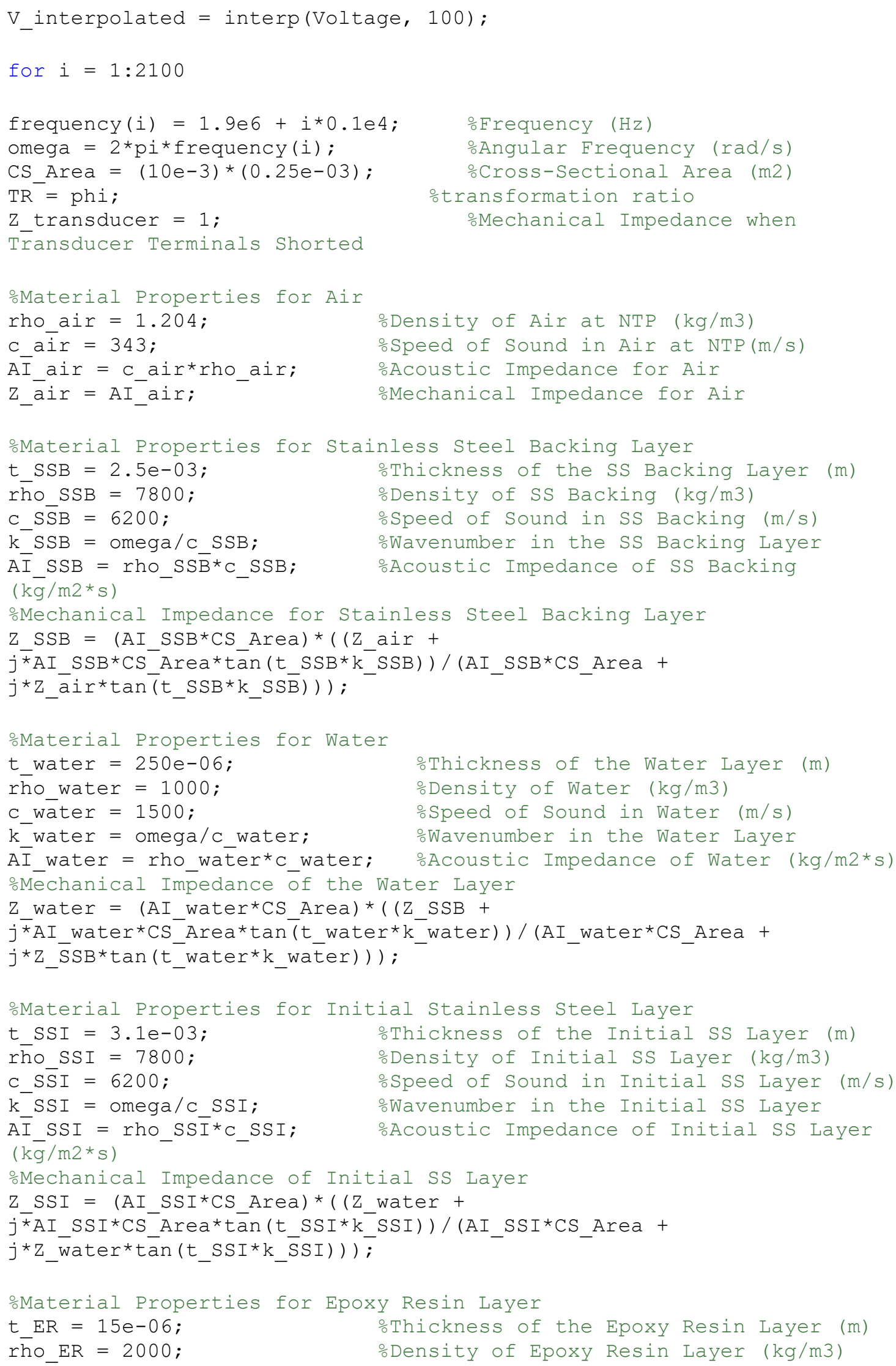




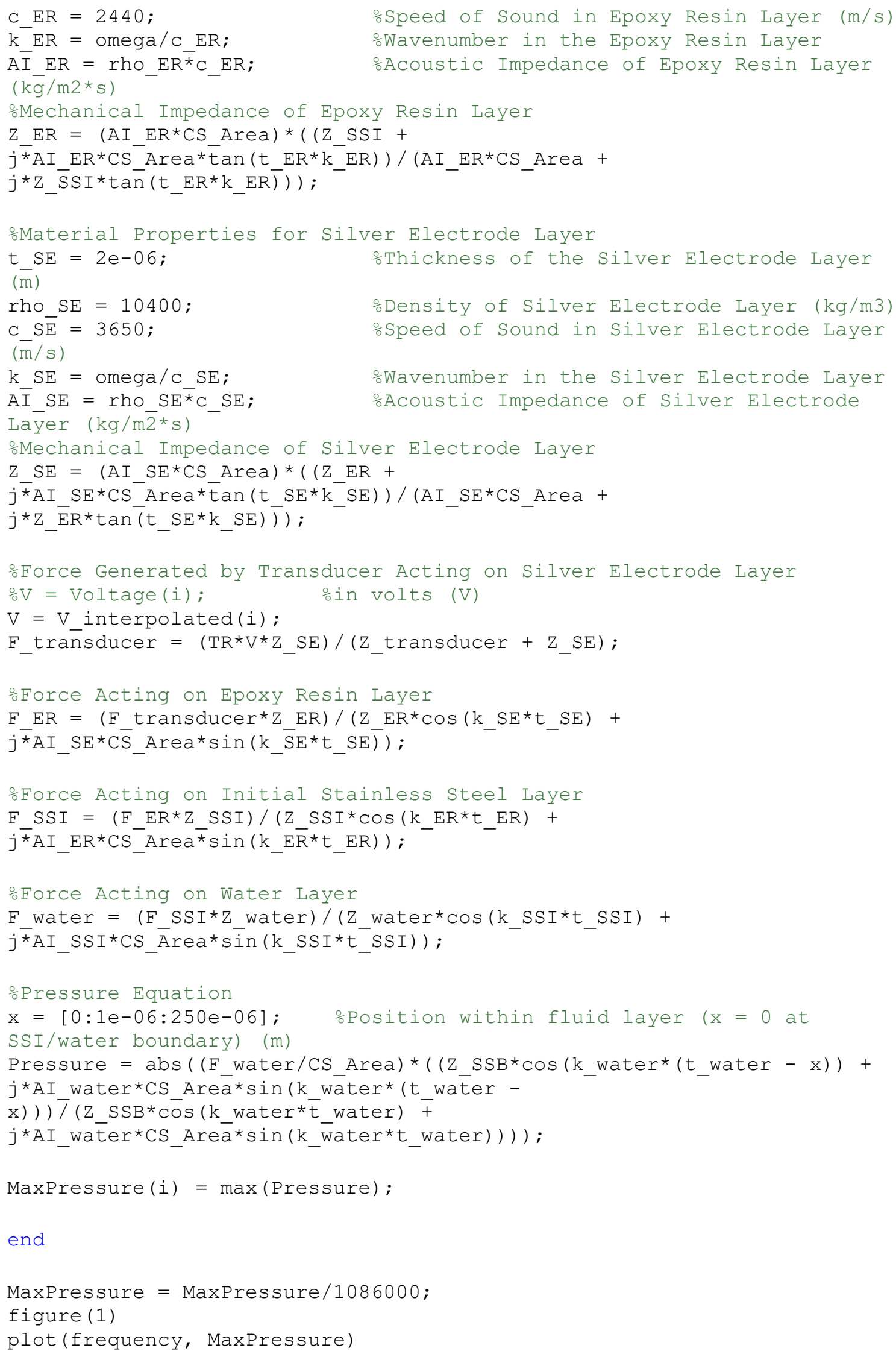


title('Maximum Pressure vs. Frequency');

xlabel ('Frequency (Hz)');

ylabel ('Normalized Max Pressure');

axis ([2e6 4 e 6 c 0 1.2] ) ;

\section{Microfluidic System Parameter Variation Code:}

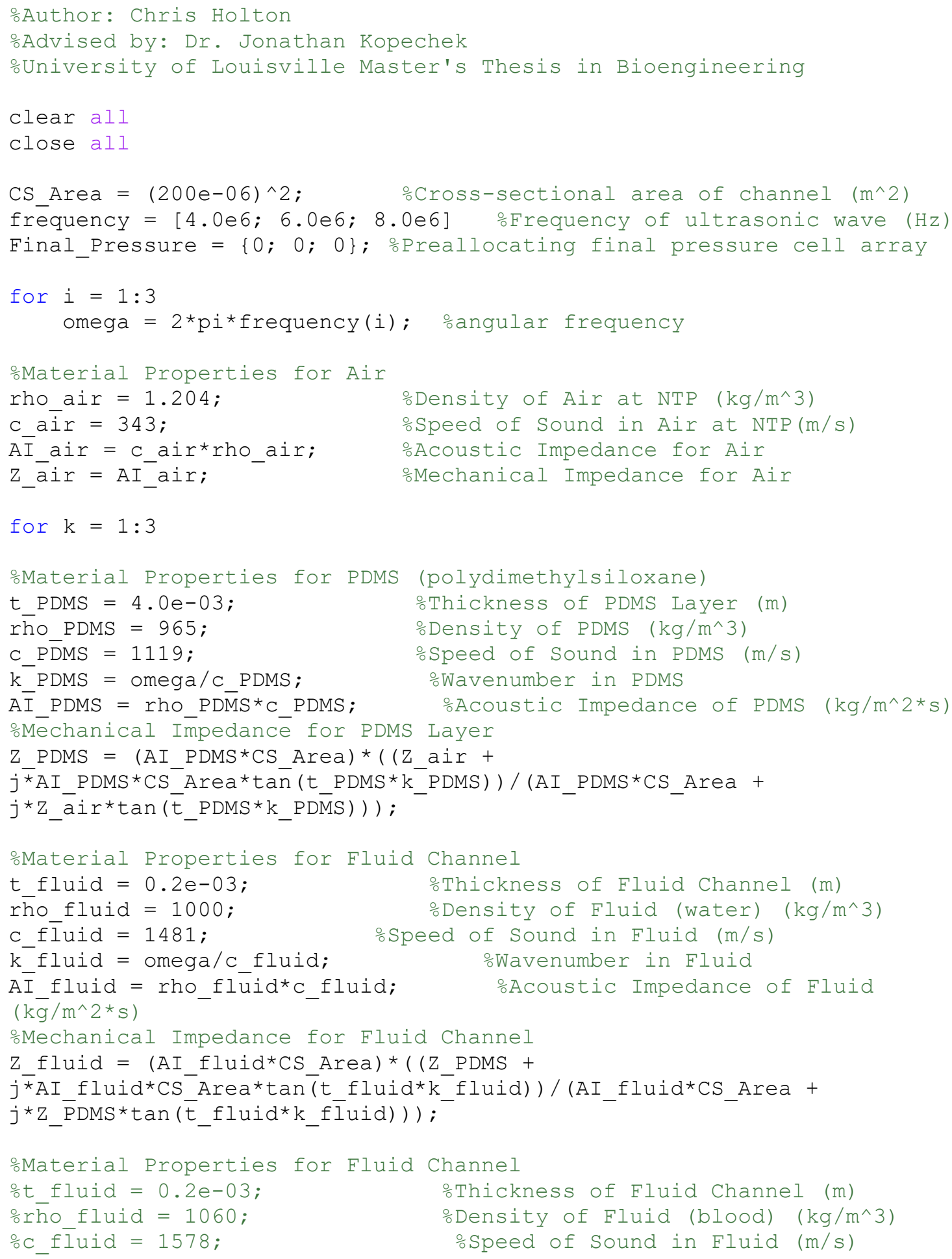




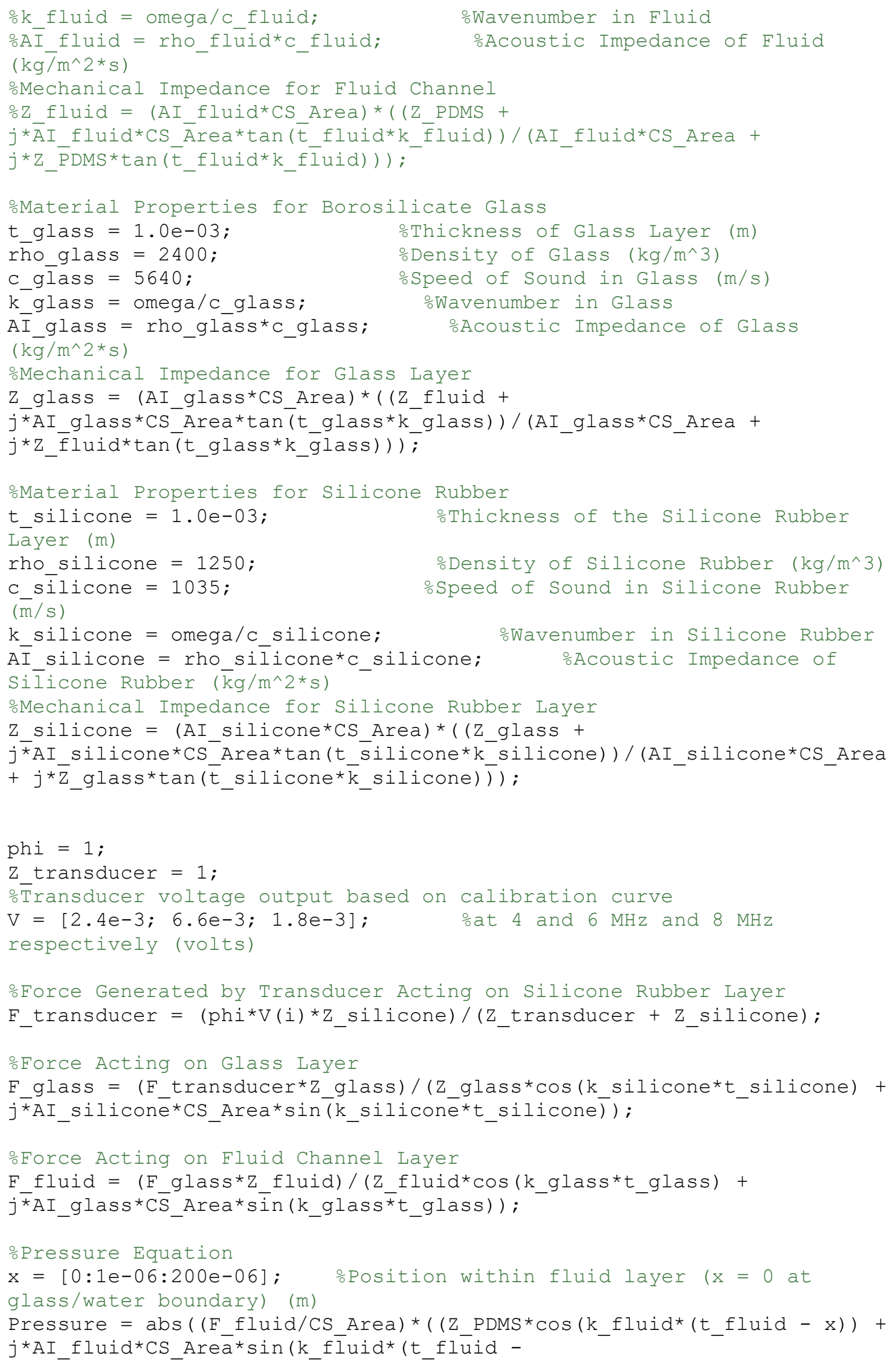




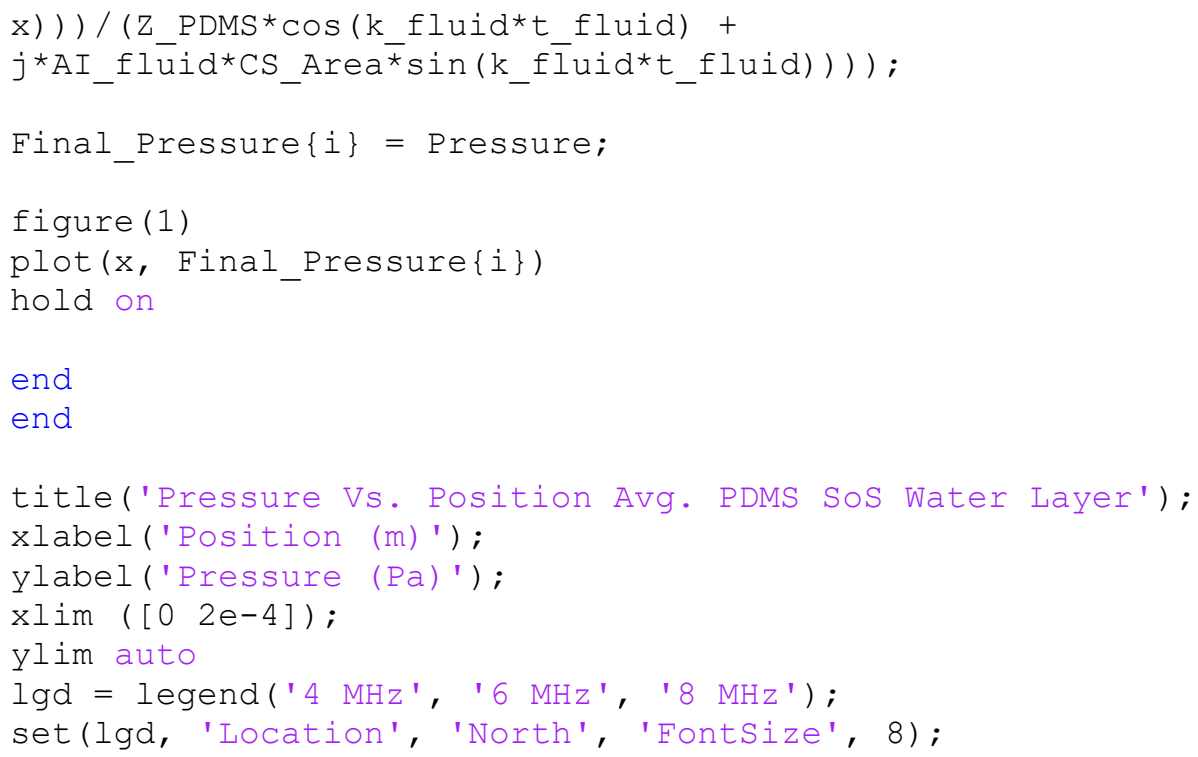

\section{Microfluidic system max pressure interpolation code:}

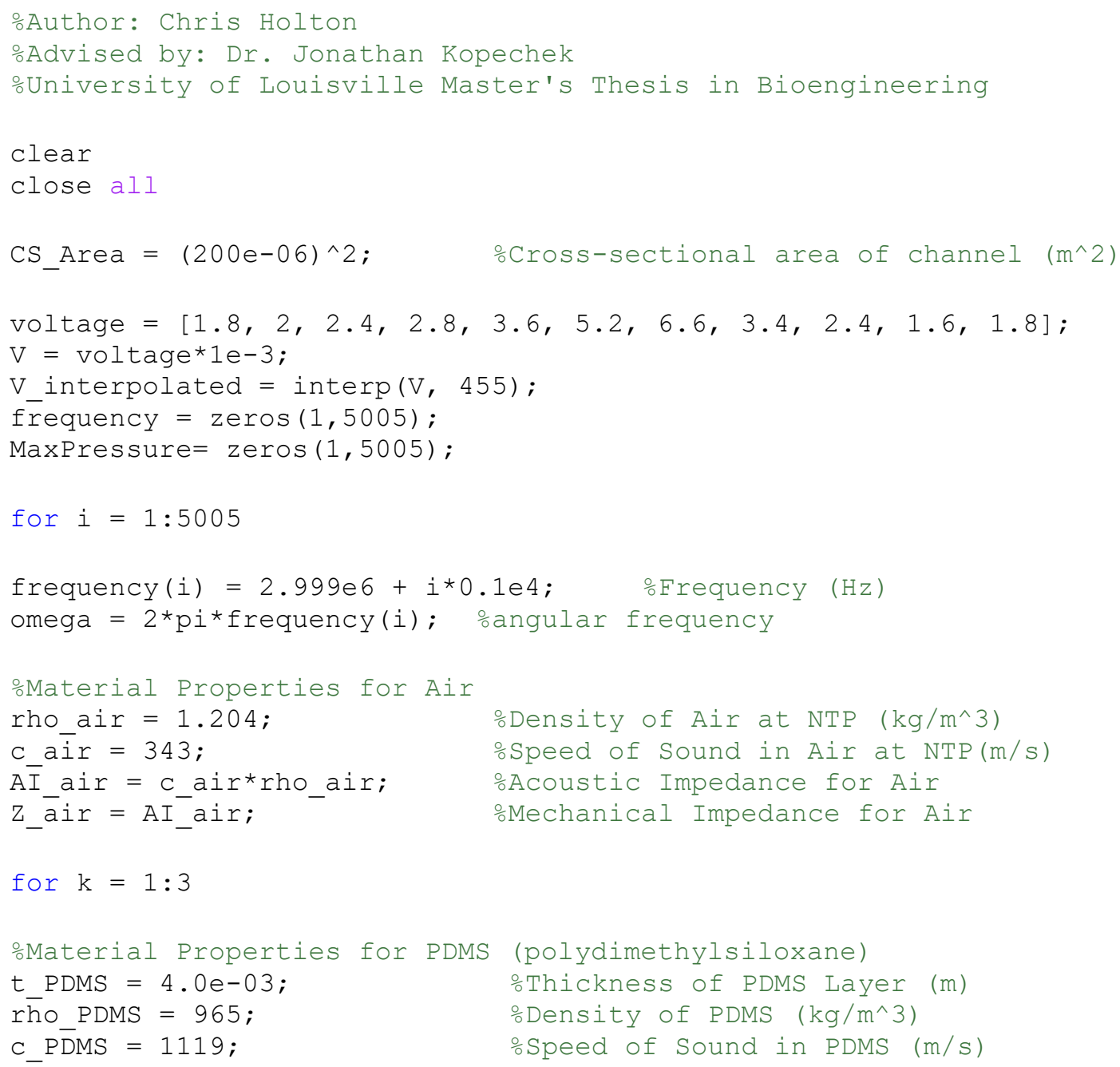




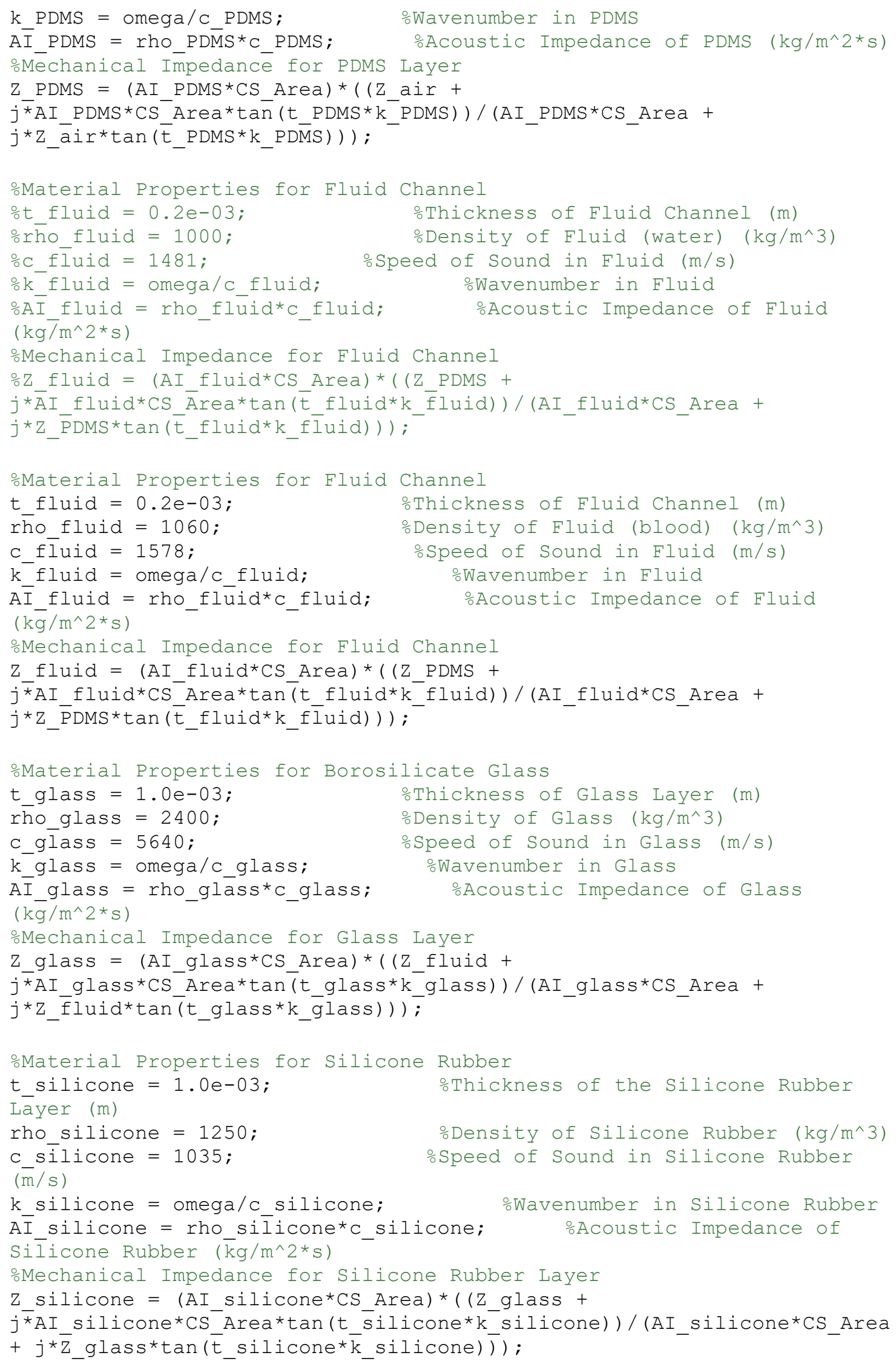




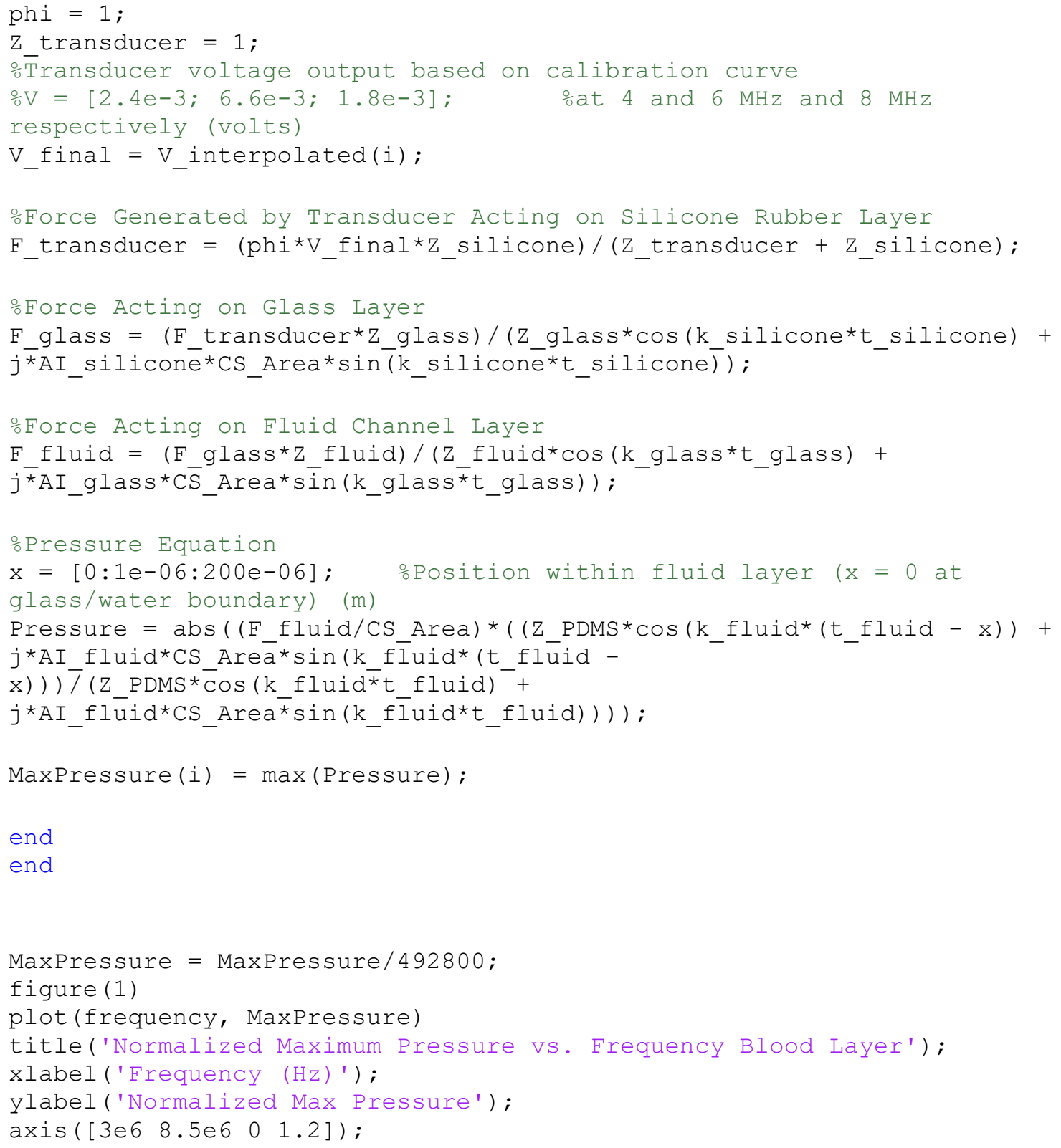




\section{CURRICULUM VITA}

Chris Holton received his B.S. in Bioengineering from the University of Louisville in May 2019. During the fall of 2017, he began his co-op placement at the Frazier Rehab Institute in Louisville, KY. There he functioned as a clinical rehabilitation engineer, assisting in the development and repair of numerous mechanical and electrical devices used for spinal cord injury rehabilitation and research. During his senior year, Chris worked with Dr. Jonathan Kopechek on developing an optimal fluid driving system for the microfluidic sonoporation system within his laboratory. For his Master's thesis, Chris worked with Dr. Kopechek again to develop a computational model characterizing the behavior of the microfluidic system to determine if its performance could be predicted for future iterations of the device. Chris graduated with his Masters in Bioengineering from the University of Louisville in May 2020 and plans to find a job in the biotech industry. 